\title{
Approximate Solutions to Ordinary Differential Equations Using Least Squares Support Vector Machines
}

\author{
Siamak Mehrkanoon, Tillmann Falck and Johan A. K. Suykens
}

\begin{abstract}
In this paper a new approach based on Least Squares Support Vector Machines (LS-SVMs) is proposed for solving linear and nonlinear ordinary differential equations (ODEs). The approximate solution is presented in closed form by means of LSSVMs, whose parameters are adjusted to minimize an appropriate error function. For the linear and nonlinear cases, these parameters are obtained by solving a system of linear and nonlinear equations respectively. The method is well suited for solving mildly stiff, nonstiff and singular ordinary differential equations with initial and boundary conditions. Numerical results demonstrate the efficiency of the proposed method over existing methods.
\end{abstract}

Index Terms-Least squares support vector machines, ordinary differential equations, closed form approximate solution, Collocation method.

\section{INTRODUCTION}

D IFFERENTIAL equations can be found in the mathematical formulation of physical phenomena in a wide variety of applications especially in science and engineering. Depending upon the form of the boundary conditions to be satisfied by the solution, problems involving ODEs can be divided into two main categories, namely initial value problems (IVPs) and boundary value problems (BVPs). Analytic solutions for these problems are not generally available and hence numerical methods must be applied.

Many methods have been developed for solving initial value problems of ODEs, such as Runge-Kutta, finite difference, predictor-corrector and collocation methods [1]-[4]. Generally speaking numerical methods for approximating the solution of the boundary value problems fall into two classes: the difference methods (e.g. shooting method) and weighted residual or series methods. In the shooting method, one tries to reduce the problem to initial value problems by providing a sufficiently good approximation of the derivative values at the initial point.

Concerning higher order ODEs, the most common approach is the reduction of the problem to a system of first-order differential equations and then solve the system by employing one of the available methods, which notably has been studied in the literature, see [2], [5], [6]. However, as some authors have remarked, this approach wastes a lot of computer time and human effort [7], [8].

Most of the traditional numerical methods provide the solution, in the form of an array, at specific preassigned mesh points

The authors are with the Department of Electrical Engineering ESATSCD-SISTA, Katholieke Universiteit Leuven, B-3001 Leuven, Belgium (email: siamak.mehrkanoon@esat.kuleuven.be; tillmann.falck@esat.kuleuven.be; johan.suykens@esat.kuleuven.be). in the domain (discrete solution) and they need an additional interpolation procedure to yield the solution for the whole domain. On the other hand in order to have an accurate solution one either has to increase the order of the method or decrease the step size. This however, increases the computational cost.

To overcome these drawbacks, attempts have been made to develop new approaches to not only solve the higher order ODEs directly without reducing it to a system of first-order differential equations, but also to provide the approximate solution in closed form (i.e. continuous and differentiable) hereby avoiding an extra interpolation procedure. One of these classes of methods is based on the use of neural network models see [9]-[15]. Lee and Kang [10] used Hopfield neural networks models to solve first order differential equations. The authors in [16] introduced a method based on feedforward neural networks to solve ordinary and partial differential equations. In that model, the approximate solution was chosen such that it, by construction, satisfied the supplementary conditions. Therefore the model function was expressed as a sum of two terms. The first term, which contains no adjustable parameters, satisfied the initial/boundary conditions and the second term involved a feedforward neural network to be trained. An unsupervised kernel least mean square algorithm is developed for solving ordinary differential equations in [17].

Despite the fact that the classical neural networks have nice properties such as universal approximation, they still suffer from having two persistent drawbacks. The first problem is the existence of many local minima solutions. The second problem is how to choose the number of hidden units.

Support Vector Machines (SVMs) are a powerful methodology for solving pattern recognition and function estimation problems [18], [19]. In this method one maps data into a high dimensional feature space and there solves a linear regression problem. It leads to solving quadratic programming problems. LS-SVMs for function estimation, classification, problems in unsupervised learning and others has been investigated in [20], [21] and [22]. In this case, the problem formulation involves equality instead of inequality constraints. The training for regression and classification problems is then done by solving a set of linear equations. It is the purpose of this paper to introduce a new approach based on LS-SVMs for solving ODEs.

The paper uses the following notation. Vector valued variables are denoted in lowercase boldface whereas variables that are neither boldfaced nor capitalized are scalar valued. Matrices are denoted in capital. Euler Script (euscript) font is used for operators. 
This paper is organized as follows. In Section II the problem statement is given. In Section III we formulate our least squares support vector machines method for the solution of linear differential equations. Section IV is devoted to the formulation of the method for nonlinear first order ODEs. Model selection and the practical implementation of the proposed method are discussed in Section V. Section VI describes the numerical experiments, discussion and comparison with other known methods.

\section{Problem statement}

This section describes the problem statement. After that, in subsection A, a short introduction to LS-SVMs for regression is given to highlight the difference to the problem considered in this paper. Finally some operators that will be used in the following sections are defined.

Consider the general $m$-th order linear ordinary differential equation with time varying coefficients of the form

$$
\mathcal{L}[y] \equiv \sum_{\ell=0}^{m} f_{\ell}(t) y^{(\ell)}(t)=r(t), \quad t \in[a, c]
$$

where $\mathcal{L}$ represents an $m$-th order linear differential operator, $[a, c]$ is the problem domain and $r(t)$ is the input signal. $f_{\ell}(t)$ are known functions and $y^{(\ell)}(t)$ denotes the $\ell$-th derivative of $y$ with respect to $t$. The $m-1$ necessary initial or boundary conditions for solving the above differential equations are:

IVP:

$$
\mathcal{J C}_{\mu}[y(t)]=p_{\mu}, \quad \mu=0, \ldots, m-1 ;
$$

BVP:

$$
\mathcal{B C}_{\mu}[y(t)]=q_{\mu}, \quad \mu=0, \ldots, m-1,
$$

where $\mathcal{J C}_{\mu}$ are the initial conditions (all constraints are applied at the same value of the independent variable i.e. $t=a$ ) and $\mathcal{B C}_{\mu}$ are the boundary conditions (the constraints are applied at multiple values of the independent variable $t$, typically at the ends of the interval $[a, c]$ in which the solution is sought). $p_{\mu}$ and $q_{\mu}$ are given scalars.

A differential equation (1) is said to be stiff when its exact solution consists of a steady state term that does not grow significantly with time, together with a transient term that decays exponentially to zero. Problems involving rapidly decaying transient solutions occur naturally in a wide variety of applications, including the study of damped mass spring system and the analysis of control systems (see [2] for more details).

If the coefficient functions $f_{\ell}(t)$ of (1) fail to be analytic at point $x=a$, then (1) is called singular ordinary differential equation.

The approaches given in [16], [17], define a trial solution to be a sum of two terms i.e. $y(t)=H(t)+F(t, N(t, P))$. The first term $H(t)$, which has to be defined by the user and in some cases is not straightforward, satisfies the initial/boundary conditions and the second term $F(t, N(t, P))$ is a singleoutput feed forward neural network with input $t$ and parameters $P$. In contrast with the approaches given in [16], [17], we build the model by incorporating the initial/boundary conditions as constraints of an optimization problem. This significantly reduces the burden placed on the user as a potentially difficult problem is handled automatically by the proposed technique.

\section{A. $L S$-SVM regression}

Let us consider a given training set $\left\{x_{i}, y_{i}\right\}_{i=1}^{N}$ with input data $x_{i} \in \mathbb{R}$ and output data $y_{i} \in \mathbb{R}$. For the purpose of this paper we only use an one-dimensional input space. The goal in a regression problem is to estimate a model of the form $y(x)=\boldsymbol{w}^{T} \boldsymbol{\varphi}(x)+b$.

The primal LS-SVM model for regression can be written as follows [21]

$$
\begin{array}{ll}
\underset{\boldsymbol{w}, b, \boldsymbol{e}}{\operatorname{minimize}} & \frac{1}{2} \boldsymbol{w}^{T} \boldsymbol{w}+\frac{\gamma}{2} \boldsymbol{e}^{T} \boldsymbol{e} \\
\text { subject to } & y_{i}=\boldsymbol{w}^{T} \boldsymbol{\varphi}\left(x_{i}\right)+b+e_{i}, \quad i=1, \ldots, N,
\end{array}
$$

where $\gamma \in \mathbb{R}^{+}, b \in \mathbb{R}, \boldsymbol{w} \in \mathbb{R}^{h} . \varphi(\cdot): \mathbb{R} \rightarrow \mathbb{R}^{h}$ is the feature map and $h$ is the dimension of the feature space. The dual solution is then given by

$$
\left[\begin{array}{c|c}
\Omega+I_{N} / \gamma & \mathbf{1}_{\boldsymbol{N}} \\
\hline \mathbf{1}_{N^{T}} & 0
\end{array}\right]\left[\begin{array}{c}
\boldsymbol{\alpha} \\
\hline b
\end{array}\right]=\left[\begin{array}{c}
\boldsymbol{y} \\
\hline 0
\end{array}\right]
$$

where $\Omega_{i j}=K\left(x_{i}, x_{j}\right)=\boldsymbol{\varphi}\left(x_{i}\right)^{T} \boldsymbol{\varphi}\left(x_{j}\right)$ is the $i j$-th entry of the positive definite kernel matrix. $\mathbf{1}_{N}=[1, \ldots, 1]^{T} \in$ $\mathbb{R}^{N}, \boldsymbol{\alpha}=\left[\alpha_{1}, \ldots, \alpha_{N}\right]^{T}, \boldsymbol{y}=\left[y_{1}, \ldots, y_{N}\right]^{T}$ and $I_{N}$ is the identity matrix. The model in the dual form becomes: $y(x)=\sum_{i=1}^{N} \alpha_{i} K\left(x, x_{i}\right)+b$. It should be noted that if $b=0$, for an explicitly known and finite dimensional feature map $\varphi$ the problem could be solved in primal (ridge regression) by eliminating $e$ and then $\boldsymbol{w}$ would be the only unknown. But in the LS-SVM approach the feature map $\varphi$ is not explicitly known in general and can be infinite dimensional. Therefore the kernel trick is used and the problem is solved in dual [20]. When we deal with differential equations, the target values $y_{i}$ are not available directly anymore so the regression approach does not directly apply. Nevertheless we can incorporate the underlying differential equation in the learning process to find an approximation for the solution.

Let us assume an explicit model $\hat{y}(t)=\boldsymbol{w}^{T} \boldsymbol{\varphi}(t)+b$ as an approximation for the solution of the differential equation. Since there are no data available in order to learn from the differential equation, we have to substitute our model into the given differential equation. Therefore we need to define the derivative of the kernel function. Making use of Mercer's Theorem [19], derivatives of the feature map can be written in terms of derivatives of the kernel function [23]. Let us define the following differential operator which will be used in subsequent sections

$$
\nabla_{n}^{m} \equiv \frac{\partial^{n+m}}{\partial u^{n} \partial v^{m}}
$$

If $\varphi(u)^{T} \boldsymbol{\varphi}(v)=K(u, v)$, then one can show that

$$
\begin{aligned}
{\left[\boldsymbol{\varphi}^{(n)}(u)\right]^{T} \boldsymbol{\varphi}^{(m)}(v) } & =\nabla_{n}^{m}\left[\boldsymbol{\varphi}(u)^{T} \boldsymbol{\varphi}(v)\right] \\
& =\nabla_{n}^{m}[K(u, v)]=\frac{\partial^{n+m} K(u, v)}{\partial u^{n} \partial v^{m}} .
\end{aligned}
$$

Using formula (4), it is possible to express all derivatives of the feature map in terms of the kernel function itself (provided that the kernel function is sufficiently differentiable). For instance 
the following relations hold,

$$
\begin{aligned}
& \nabla_{1}^{0}[K(u, v)]=\frac{\partial\left(\boldsymbol{\varphi}(u)^{T} \boldsymbol{\varphi}(v)\right)}{\partial u}=\boldsymbol{\varphi}^{(1)}(u)^{T} \boldsymbol{\varphi}(v), \\
& \nabla_{0}^{1}[K(u, v)]=\frac{\partial\left(\boldsymbol{\varphi}(u)^{T} \boldsymbol{\varphi}(v)\right)}{\partial v}=\boldsymbol{\varphi}(u)^{T} \boldsymbol{\varphi}^{(1)}(v), \\
& \nabla_{2}^{0}[K(u, v)]=\frac{\partial^{2}\left(\boldsymbol{\varphi}(u)^{T} \boldsymbol{\varphi}(v)\right)}{\partial u^{2}}=\boldsymbol{\varphi}^{(2)}(u)^{T} \boldsymbol{\varphi}(v) .
\end{aligned}
$$

\section{Formulation OF THE METHOD FOR THE LINEAR ODE CASE}

Let us assume that a general approximate solution to (1) is of the form of $\hat{y}(t)=\boldsymbol{w}^{T} \boldsymbol{\varphi}(t)+b$, where $\boldsymbol{w}$ and $b$ are unknowns of the model that have to be determined. To obtain the optimal value of these parameters, collocation methods can be used [24] which assume a discretization of the interval $[a, c]$ into a set of collocation points $\Upsilon=\left\{a=t_{1}<t_{2}<\ldots<t_{N}=c\right\}$. Therefore the $\boldsymbol{w}$ and $b$ are to be found by solving the following optimization problem:

\section{For the IVP Case:}

$$
\begin{array}{ll}
\underset{\hat{y}}{\operatorname{minimize}} & \frac{1}{2} \sum_{i=1}^{N}\left[(\mathcal{L}[\hat{y}]-r)\left(t_{i}\right)\right]^{2} \\
\text { subject to } & \mathcal{J C}_{\mu}[\hat{y}(t)]=p_{\mu}, \quad \mu=0, \ldots, m-1 .
\end{array}
$$

\section{For the BVP case:}

$$
\begin{array}{ll}
\underset{\hat{y}}{\operatorname{minimize}} & \frac{1}{2} \sum_{i=1}^{N}\left[(\mathcal{L}[\hat{y}]-r)\left(t_{i}\right)\right]^{2} \\
\text { subject to } & \mathcal{B C}_{\mu}[\hat{y}(t)]=q_{\mu}, \quad \mu=0, \ldots, m-1,
\end{array}
$$

where $N$ is the number of collocation points (which is equal to the number of training points) used to undertake the learning process. In what follows we formulate the optimization problem in the LS-SVM framework for solving linear ordinary differential equations. For notational convenience let us list the following notations which are used in the following sections,

$$
\begin{aligned}
& {\left[\nabla_{n}^{m} K\right](t, s)=\left.\left[\nabla_{n}^{m} K(u, v)\right]\right|_{u=t, v=s},} \\
& {\left[\Omega_{n}^{m}\right]_{i, j}=\left.\nabla_{n}^{m}[K(u, v)]\right|_{u=t_{i}, v=t_{j}}=\left.\frac{\partial^{n+m} K(u, v)}{\partial u^{n} \partial v^{m}}\right|_{u=t_{i}, v=t_{j}},} \\
& {\left[\Omega_{0}^{0}\right]_{i, j}=\left.\nabla_{0}^{0}[K(u, v)]\right|_{u=t_{i}, v=t_{j}}=K\left(t_{i}, t_{j}\right) .}
\end{aligned}
$$

Where $\left[\Omega_{n}^{m}\right]_{i, j}$ denotes the $(i, j)$-th entry of matrix $\Omega_{n}^{m}$. The notation $M_{k: l, m: n}$ is used for selecting a submatrix of matrix $M$ consisting of rows $k$ to $l$ and columns $m$ to $n$. $M_{i, \text { : denotes }}$ the $i$-th row of matrix $M . M_{:, j}$ denotes the $j$-th column of matrix $M$.

\section{A. First order IVP}

As a first example consider the following first order initial value problem,

$$
y^{\prime}(t)-f_{1}(t) y(t)=r(t), \quad y(a)=p_{1}, \quad a \leq t \leq c .
$$

In the LS-SVM framework the approximate solution can be obtained by solving the following optimization problem,

$$
\begin{array}{ll}
\underset{\boldsymbol{w}, b, \boldsymbol{e}}{\operatorname{minimize}} & \frac{1}{2} \boldsymbol{w}^{T} \boldsymbol{w}+\frac{\gamma}{2} \boldsymbol{e}^{T} \boldsymbol{e} \\
\text { subject to } & \boldsymbol{w}^{T} \boldsymbol{\varphi}^{\prime}\left(t_{i}\right)=f_{1}\left(t_{i}\right)\left[\boldsymbol{w}^{T} \boldsymbol{\varphi}\left(t_{i}\right)+b\right]+ \\
& r\left(t_{i}\right)+e_{i}, \quad i=2, \ldots, N, \\
& \boldsymbol{w}^{T} \boldsymbol{\varphi}\left(t_{1}\right)+b=p_{1} .
\end{array}
$$

This problem is obtained by combining the LS-SVM cost function with constraints constructed by imposing the approximate solution $\hat{y}(t)=\boldsymbol{w}^{T} \boldsymbol{\varphi}(t)+b$, given by the LS-SVM model, to satisfy the given differential equation with corresponding initial condition at collocation points $\left\{t_{i}\right\}_{i=1}^{N}$. Problem (8) is a quadratic minimization under linear equality constraints, which enables an efficient solution.

Lemma III.1. Given a positive definite kernel function $K$ : $\mathbb{R} \times \mathbb{R} \rightarrow \mathbb{R}$ with $K(t, s)=\varphi(t)^{T} \varphi(s)$ and a regularization constant $\gamma \in \mathbb{R}^{+}$, the solution to (8) is obtained by solving the following dual problem:

$$
\left[\begin{array}{c|c|c}
\mathcal{K}+I_{N-1} / \gamma & \boldsymbol{h}_{\boldsymbol{p}_{\mathbf{1}}} & -\boldsymbol{f}_{\mathbf{1}} \\
\hline \boldsymbol{h}_{\boldsymbol{p}_{\mathbf{1}}}{ }^{T} & 1 & 1 \\
\hline-\boldsymbol{f}_{\mathbf{1}}{ }^{T} & 1 & 0
\end{array}\right]\left[\begin{array}{c}
\boldsymbol{\alpha} \\
\hline \beta \\
\hline b
\end{array}\right]=\left[\begin{array}{c}
\boldsymbol{r} \\
\hline p_{1} \\
\hline 0
\end{array}\right]
$$

with

$$
\begin{aligned}
& \boldsymbol{\alpha}=\left[\alpha_{2}, \ldots, \alpha_{N}\right]^{T}, \boldsymbol{f}_{\mathbf{1}}=\left[f_{1}\left(t_{2}\right), \ldots, f_{1}\left(t_{N}\right)\right]^{T} \in \mathbb{R}^{N-1}, \\
& \boldsymbol{r}=\left[r\left(t_{2}\right), \ldots, r\left(t_{N}\right)\right]^{T} \in \mathbb{R}^{N-1}, \\
& \mathcal{K}=\tilde{\Omega}_{1}^{1}-D_{1} \tilde{\Omega}_{1}^{0}-\tilde{\Omega}_{0}^{1} D_{1}+D_{1} \tilde{\Omega}_{0}^{0} D_{1}, \\
& \boldsymbol{h}_{\boldsymbol{p}_{\mathbf{1}}}=\left[\Omega_{0}^{1}\right]_{1,2: N}^{T}-D_{1}\left[\Omega_{0}^{0}\right]_{1,2: N}^{T} .
\end{aligned}
$$

$D_{1}$ is a diagonal matrix with the elements of $f_{1}$ on the main diagonal. $\left[\Omega_{n}^{m}\right]_{1,2: N}=\left[\left[\Omega_{n}^{m}\right]_{1,2}, \ldots,\left[\Omega_{n}^{m}\right]_{1, N}\right]$ and $\tilde{\Omega}_{n}^{m}=$ $\left[\Omega_{n}^{m}\right]_{2: N, 2: N}$ for $n, m=0,1$. Also note that $\mathcal{K} \in \mathbb{R}^{(N-1) \times(N-1)}$ and $\boldsymbol{h}_{\boldsymbol{p}_{1}} \in \mathbb{R}^{N-1}$.

Proof: The Lagrangian of the constrained optimization problem (8) becomes

$$
\begin{aligned}
& \mathcal{L}\left(\boldsymbol{w}, b, e_{i}, \alpha_{i}, \beta\right)= \\
& \frac{1}{2} \boldsymbol{w}^{T} \boldsymbol{w}+\frac{\gamma}{2} \boldsymbol{e}^{T} \boldsymbol{e}-\sum_{i=2}^{N} \alpha_{i}\left[\boldsymbol{w}^{T}\left(\boldsymbol{\varphi}^{\prime}\left(t_{i}\right)-f_{1}\left(t_{i}\right) \boldsymbol{\varphi}\left(t_{i}\right)\right)\right. \\
& \left.-f_{1}\left(t_{i}\right) b-r_{i}-e_{i}\right]-\beta\left(\boldsymbol{w}^{T} \boldsymbol{\varphi}\left(t_{1}\right)+b-p_{1}\right)
\end{aligned}
$$

where $\left\{\alpha_{i}\right\}_{i=2}^{N}$ and $\beta$ are Lagrange multipliers and $r_{i}=r\left(t_{i}\right)$ for $i=2, \ldots, N$. Then the Karush-Kuhn-Tucker (KKT) optimality conditions are as follows,

$$
\begin{aligned}
& \frac{\partial \mathcal{L}}{\partial \boldsymbol{w}}=0 \rightarrow \boldsymbol{w}=\sum_{i=2}^{N} \alpha_{i}\left(\boldsymbol{\varphi}^{\prime}\left(t_{i}\right)-f_{1}\left(t_{i}\right) \boldsymbol{\varphi}\left(t_{i}\right)\right)+ \\
& \beta \boldsymbol{\varphi}\left(t_{1}\right), \frac{\partial \mathcal{L}}{\partial b}=0 \rightarrow \sum_{i=2}^{N} \alpha_{i} f_{1}\left(t_{i}\right)-\beta=0, \frac{\partial \mathcal{L}}{\partial e_{i}}=0 \rightarrow e_{i}= \\
& -\frac{\alpha_{i}}{\gamma}, \quad i=2, \ldots, N, \frac{\partial \mathcal{L}}{\partial \alpha_{i}}=0 \rightarrow \boldsymbol{w}^{T}\left(\varphi^{\prime}\left(t_{i}\right)-f_{1}\left(t_{i}\right) \boldsymbol{\varphi}\left(t_{i}\right)\right)- \\
& f_{1}\left(t_{i}\right) b-e_{i}=r_{i}, \quad i=2, \ldots, N, \frac{\partial \mathcal{L}}{\partial \beta}=0 \rightarrow \boldsymbol{w}^{T} \boldsymbol{\varphi}\left(t_{1}\right)+b=p_{1} .
\end{aligned}
$$


After elimination of the primal variables $\boldsymbol{w}$ and $\left\{e_{i}\right\}_{i=2}^{N}$ and making use of Mercer's Theorem, the solution is given in the dual by

$$
\left\{\begin{aligned}
r_{i}= & \sum_{j=2}^{N} \alpha_{j}\left[\left[\Omega_{1}^{1}\right]_{j, i}-f_{1}\left(t_{i}\right)\left(\left[\Omega_{1}^{0}\right]_{j, i}-f_{1}\left(t_{j}\right)\left[\Omega_{0}^{0}\right]_{j, i}\right)\right. \\
& \left.-f_{1}\left(t_{j}\right)\left[\Omega_{0}^{1}\right]_{j, i}\right]+\beta\left(\left[\Omega_{0}^{1}\right]_{1, i}-f_{1}\left(t_{i}\right)\left[\Omega_{0}^{0}\right]_{1, i}\right) \\
& +\frac{\alpha_{i}}{\gamma}-f_{1}\left(t_{i}\right) b, \quad i=2, \ldots, N, \\
p_{1}= & \sum_{j=2}^{N} \alpha_{j}\left(\left[\Omega_{1}^{0}\right]_{j, 1}-f_{1}\left(t_{j}\right)\left[\Omega_{0}^{0}\right]_{j, 1}\right)+\beta\left[\Omega_{0}^{0}\right]_{1,1}+b, \\
0= & \sum_{j=2}^{N} \alpha_{j} f_{1}\left(t_{j}\right)-\beta
\end{aligned}\right.
$$

and writing these equations in matrix form gives the linear system in (9).

The model in the dual form becomes

$$
\begin{aligned}
\hat{y}(t)= & \sum_{i=2}^{N} \alpha_{i}\left(\left[\nabla_{1}^{0} K\right]\left(t_{i}, t\right)-f_{1}\left(t_{i}\right)\left[\nabla_{0}^{0} K\right]\left(t_{i}, t\right)\right) \\
& +\beta\left[\nabla_{0}^{0} K\right]\left(t_{1}, t\right)+b
\end{aligned}
$$

where $K$ is the kernel function.

\section{B. Second order IVP and BVP}

\section{IVP case:}

Let us consider a second order IVP of the form,

$$
\begin{aligned}
& y^{\prime \prime}(t)=f_{1}(t) y^{\prime}(t)+f_{2}(t) y(t)+r(t), \quad t \in[a, c] \\
& y(a)=p_{1}, \quad y^{\prime}(a)=p_{2} .
\end{aligned}
$$

The approximate solution, $\hat{y}(t)=\boldsymbol{w}^{T} \boldsymbol{\varphi}(t)+b$, is then obtained by solving the following optimization problem,

$$
\begin{array}{cl}
\underset{\boldsymbol{w}, b, \boldsymbol{e}}{\operatorname{minimize}} & \frac{1}{2} \boldsymbol{w}^{T} \boldsymbol{w}+\frac{\gamma}{2} \boldsymbol{e}^{T} \boldsymbol{e} \\
\text { subject to } & \boldsymbol{w}^{T} \boldsymbol{\varphi}^{\prime \prime}\left(t_{i}\right)=f_{1}\left(t_{i}\right) \boldsymbol{w}^{T} \boldsymbol{\varphi}^{\prime}\left(t_{i}\right)+ \\
& f_{2}\left(t_{i}\right)\left[\boldsymbol{w}^{T} \boldsymbol{\varphi}\left(t_{i}\right)+b\right]+r\left(t_{i}\right)+e_{i}, \quad i=2, \ldots, N \\
& \boldsymbol{w}^{T} \boldsymbol{\varphi}\left(t_{1}\right)+b=p_{1}, \\
& \boldsymbol{w}^{T} \boldsymbol{\varphi}^{\prime}\left(t_{1}\right)=p_{2} .
\end{array}
$$

Lemma III.2. Given a positive definite kernel function $K$ : $\mathbb{R} \times \mathbb{R} \rightarrow \mathbb{R}$ with $K(t, s)=\boldsymbol{\varphi}(t)^{T} \boldsymbol{\varphi}(s)$ and a regularization constant $\gamma \in \mathbb{R}^{+}$, the solution to (11) is obtained by solving the following dual problem:

$\left[\begin{array}{c|c|c|c}\mathcal{K}+I_{N-1} / \gamma & \boldsymbol{h}_{\boldsymbol{p}_{\mathbf{1}}} & \boldsymbol{h}_{\boldsymbol{p}_{\mathbf{2}}} & -\boldsymbol{f}_{\mathbf{2}} \\ \hline \boldsymbol{h}_{\boldsymbol{p}_{\mathbf{1}}}{ }^{T} & 1 & 0 & 1 \\ \hline \boldsymbol{h}_{\boldsymbol{p}_{\mathbf{2}}{ }^{T}} & 0 & {\left[\Omega_{1}^{1}\right]_{1,1}} & 0 \\ \hline-\boldsymbol{f}_{\mathbf{2}}{ }^{T} & 1 & 0 & 0\end{array}\right]\left[\begin{array}{c}\boldsymbol{\alpha} \\ \hline \beta_{1} \\ \hline \beta_{2} \\ \hline b\end{array}\right]=\left[\begin{array}{c}\boldsymbol{r} \\ \hline p_{1} \\ \hline p_{2} \\ \hline 0\end{array}\right]$

where

$$
\begin{aligned}
& \boldsymbol{\alpha}=\left[\alpha_{2}, \ldots, \alpha_{N}\right]^{T}, \boldsymbol{f}_{\mathbf{1}}=\left[f_{1}\left(t_{2}\right), \ldots, f_{1}\left(t_{N}\right)\right]^{T} \in \mathbb{R}^{N-1}, \\
& \boldsymbol{f}_{\mathbf{2}}=\left[f_{2}\left(t_{2}\right), \ldots, f_{2}\left(t_{N}\right)\right]^{T} \in \mathbb{R}^{N-1}, \\
& \boldsymbol{r}=\left[r\left(t_{2}\right), \ldots, r\left(t_{N}\right)\right]^{T} \in \mathbb{R}^{N-1}, \\
& \mathcal{K}=\tilde{\Omega}_{2}^{2}-D_{1} \tilde{\Omega}_{2}^{1}-D_{2} \tilde{\Omega}_{2}^{0}-\tilde{\Omega}_{1}^{2} D_{1}-\tilde{\Omega}_{0}^{2} D_{2} \\
& \quad+D_{1} \tilde{\Omega}_{1}^{1} D_{1}+D_{1} \tilde{\Omega}_{0}^{1} D_{2}+D_{2} \tilde{\Omega}_{1}^{0} D_{1}+D_{2} \tilde{\Omega}_{0}^{0} D_{2}, \\
& \boldsymbol{h}_{p_{1}}=\left[\Omega_{0}^{2}\right]_{1,2: N}^{T}-D_{1}\left[\Omega_{0}^{1}\right]_{1,2: N}^{T}-D_{2}\left[\Omega_{0}^{0}\right]_{1,2: N}^{T}, \\
& \boldsymbol{h}_{p_{2}}=\left[\Omega_{1}^{2}\right]_{1,2: N}^{T}-D_{1}\left[\Omega_{1}^{1}\right]_{1,2: N}^{T}-D_{2}\left[\Omega_{1}^{0}\right]_{1,2: N}^{T} .
\end{aligned}
$$

$D_{1}$ and $D_{2}$ are diagonal matrices with the elements of $\boldsymbol{f}_{1}$ and $\boldsymbol{f}_{\mathbf{2}}$ on the main diagonal respectively. Note that $\mathcal{K} \in \mathbb{R}^{(N-1) \times(N-1)}$ and $\boldsymbol{h}_{p_{1}}, \boldsymbol{h}_{p_{2}} \in \mathbb{R}^{N-1}$. $\left[\Omega_{n}^{m}\right]_{1,2: N}=$ $\left[\left[\Omega_{n}^{m}\right]_{1,2}, \ldots,\left[\Omega_{n}^{m}\right]_{1, N}\right]$ for $n=0,1$ and $m=0,1,2$. $\tilde{\Omega}_{n}^{m}=$ $\left[\Omega_{n}^{m}\right]_{2: N, 2: N}$ for $m, n=0,1,2$.

Proof: Consider the Lagrangian of problem (11):

$$
\begin{aligned}
& \mathcal{L}\left(\boldsymbol{w}, b, e_{i}, \alpha_{i}, \beta_{1}, \beta_{2}\right)= \\
& \frac{1}{2} \boldsymbol{w}^{T} \boldsymbol{w}+\frac{\gamma}{2} \boldsymbol{e}^{T} \boldsymbol{e}-\sum_{i=2}^{N} \alpha_{i}\left[\boldsymbol { w } ^ { T } \left(\boldsymbol{\varphi}^{\prime \prime}\left(t_{i}\right)-f_{1}\left(t_{i}\right) \boldsymbol{\varphi}^{\prime}\left(t_{i}\right)-\right.\right. \\
& \left.\left.f_{2}\left(t_{i}\right) \boldsymbol{\varphi}\left(t_{i}\right)\right)-f_{2}\left(t_{i}\right) b-r_{i}-e_{i}\right]-\beta_{1}\left(\boldsymbol{w}^{T} \boldsymbol{\varphi}\left(t_{1}\right)+b-p_{1}\right) \\
& -\beta_{2}\left(\boldsymbol{w}^{T} \boldsymbol{\varphi}^{\prime}\left(t_{1}\right)-p_{2}\right)
\end{aligned}
$$

where $\left\{\alpha_{i}\right\}_{i=2}^{N}, \beta_{1}$ and $\beta_{2}$ are Lagrange multipliers. The Karush-Kuhn-Tucker (KKT) optimality conditions are as follows,

$\frac{\partial \mathcal{L}}{\partial \boldsymbol{w}}=0 \rightarrow \boldsymbol{w}=\sum_{i=2}^{N} \alpha_{i}\left(\boldsymbol{\varphi}_{i}^{\prime \prime}-f_{1}\left(t_{i}\right) \boldsymbol{\varphi}_{i}^{\prime}-f_{2}\left(t_{i}\right) \boldsymbol{\varphi}_{i}\right)+$ $\beta_{1} \varphi_{1}+\beta_{2} \varphi_{1}^{\prime}, \frac{\partial \mathcal{L}}{\partial b}=0 \rightarrow \sum_{i=2}^{N} \alpha_{i} f_{2}\left(t_{i}\right)-\beta_{1}=0, \frac{\partial \mathcal{L}}{\partial e_{i}}=0 \rightarrow$ $e_{i}=-\frac{\alpha_{i}}{\gamma}, \quad i=2, \ldots, N, \frac{\partial \mathcal{L}}{\partial \alpha_{i}}=0 \rightarrow \boldsymbol{w}^{T}\left(\varphi_{i}^{\prime \prime}-f_{1}\left(t_{i}\right) \boldsymbol{\varphi}_{i}^{\prime}-\right.$ $\left.f_{2}\left(t_{i}\right) \varphi_{i}\right)-f_{2}\left(t_{i}\right) b-e_{i}=r_{i}, \quad i=2, \ldots, N, \frac{\partial \mathcal{L}}{\partial \beta_{1}}=0 \rightarrow$ $\boldsymbol{w}^{T} \boldsymbol{\varphi}_{1}+b=p_{1}, \frac{\partial \mathcal{L}}{\partial \beta_{2}}=0 \rightarrow \boldsymbol{w}^{T} \boldsymbol{\varphi}_{1}^{\prime}=p_{2}$, where $\boldsymbol{\varphi}_{i}=\boldsymbol{\varphi}\left(t_{i}\right)$, $\varphi_{i}^{\prime}=\varphi^{\prime}\left(t_{i}\right)$ and $\varphi_{i}^{\prime \prime}=\varphi^{\prime \prime}\left(t_{i}\right)$ for $i=1, \ldots, N$.

Applying the kernel trick and eliminating $\boldsymbol{w}$ and $\left\{e_{i}\right\}_{i=2}^{N}$ leads to

$$
\left\{\begin{aligned}
r_{i}= & \sum_{j=2}^{N} \alpha_{j}\left[\left[\Omega_{2}^{2}\right]_{j, i}\right. \\
& -f_{1}\left(t_{i}\right)\left(\left[\Omega_{2}^{1}\right]_{j, i}-f_{1}\left(t_{j}\right)\left[\Omega_{1}^{1}\right]_{j, i}-f_{2}\left(t_{j}\right)\left[\Omega_{0}^{1}\right]_{j, i}\right) \\
& -f_{2}\left(t_{i}\right)\left(\left[\Omega_{2}^{0}\right]_{j, i}-f_{1}\left(t_{j}\right)\left[\Omega_{1}^{0}\right]_{j, i}-f_{2}\left(t_{j}\right)\left[\Omega_{0}^{0}\right]_{j, i}\right) \\
& \left.-f_{1}\left(t_{j}\right)\left[\Omega_{1}^{2}\right]_{j, i}-f_{2}\left(t_{j}\right)\left[\Omega_{0}^{2}\right]_{j, i}\right] \\
& +\beta_{1}\left(\left[\Omega_{0}^{2}\right]_{1, i}-f_{1}\left(t_{i}\right)\left[\Omega_{0}^{1}\right]_{1, i}-f_{2}\left(t_{i}\right)\left[\Omega_{0}^{0}\right]_{1, i}\right) \\
& +\beta_{2}\left(\left[\Omega_{1}^{2}\right]_{1, i}-f_{1}\left(t_{i}\right)\left[\Omega_{1}^{1}\right]_{1, i}-f_{2}\left(t_{i}\right)\left[\Omega_{1}^{0}\right]_{1, i}\right) \\
& +\frac{\alpha_{i}}{\gamma}-f_{2}\left(t_{i}\right) b \quad, \quad i=2, \ldots, N,
\end{aligned}\right.
$$




$$
\left\{\begin{aligned}
p_{1} & =\sum_{j=2}^{N} \alpha_{j}\left(\left[\Omega_{2}^{0}\right]_{j, 1}-f_{1}\left(t_{j}\right)\left[\Omega_{1}^{0}\right]_{j, 1}-f_{2}\left(t_{j}\right)\left[\Omega_{0}^{0}\right]_{j, 1}\right) \\
& +\beta_{1}\left[\Omega_{0}^{0}\right]_{1,1}+\beta_{2}\left[\Omega_{1}^{0}\right]_{1,1}+b \\
p_{2}= & \sum_{j=2}^{N} \alpha_{j}\left(\left[\Omega_{2}^{1}\right]_{j, 1}-f_{1}\left(t_{j}\right)\left[\Omega_{1}^{1}\right]_{j, 1}-f_{2}\left(t_{j}\right)\left[\Omega_{0}^{1}\right]_{j, 1}\right) \\
& +\beta_{1}\left[\Omega_{0}^{1}\right]_{1,1}+\beta_{2}\left[\Omega_{1}^{1}\right]_{1,1}, \\
0= & \sum_{j=2}^{N} \alpha_{j} f_{2}\left(t_{j}\right)-\beta_{1} .
\end{aligned}\right.
$$

Finally writing these equations in matrix form will result in the linear system (12).

The LS-SVM model for the solution and its derivative in the dual form become:

$$
\begin{aligned}
\hat{y}(t)= & \sum_{i=2}^{N} \alpha_{i}\left(\left[\nabla_{2}^{0} K\right]\left(t_{i}, t\right)-f_{1}\left(t_{i}\right)\left[\nabla_{1}^{0} K\right]\left(t_{i}, t\right)-\right. \\
& \left.f_{2}\left(t_{i}\right)\left[\nabla_{0}^{0} K\right]\left(t_{i}, t\right)\right)+\beta_{1}\left[\nabla_{0}^{0} K\right]\left(t_{1}, t\right)+ \\
& \beta_{2}\left[\nabla_{1}^{0} K\right]\left(t_{1}, t\right)+b, \\
\frac{d \hat{y}(t)}{d t}= & \sum_{i=2}^{N} \alpha_{i}\left(\left[\nabla_{2}^{1} K\right]\left(t_{i}, t\right)-f_{1}\left(t_{i}\right)\left[\nabla_{1}^{1} K\right]\left(t_{i}, t\right)-\right. \\
& \left.f_{2}\left(t_{i}\right)\left[\nabla_{0}^{1} K\right]\left(t_{i}, t\right)\right)+\beta_{1}\left[\nabla_{0}^{1} K\right]\left(t_{1}, t\right)+ \\
& \beta_{2}\left[\nabla_{1}^{1} K\right]\left(t_{1}, t\right) .
\end{aligned}
$$

\section{BVP case:}

Consider the second order boundary value problem of ODEs of the form

$$
\begin{aligned}
& y^{\prime \prime}(t)=f_{1}(t) y^{\prime}(t)+f_{2}(t) y(t)+r(t), \quad t \in[a, c] \\
& y(a)=p_{1}, \quad y(c)=q_{1} .
\end{aligned}
$$

Then the parameters of the closed form approximation of the solution can be obtained by solving the following optimization problem

$$
\begin{array}{cl}
\underset{\boldsymbol{w}, b, \boldsymbol{e}}{\operatorname{minimize}} & \frac{1}{2} \boldsymbol{w}^{T} \boldsymbol{w}+\frac{\gamma}{2} \boldsymbol{e}^{T} \boldsymbol{e} \\
\text { subject to } & \boldsymbol{w}^{T} \boldsymbol{\varphi}^{\prime \prime}\left(t_{i}\right)=f_{1}\left(t_{i}\right) \boldsymbol{w}^{T} \boldsymbol{\varphi}^{\prime}\left(t_{i}\right)+ \\
& f_{2}\left(t_{i}\right)\left[\boldsymbol{w}^{T} \boldsymbol{\varphi}\left(t_{i}\right)+b\right]+r\left(t_{i}\right)+e_{i}, \quad i=2, \ldots, N-1, \\
& \boldsymbol{w}^{T} \boldsymbol{\varphi}\left(t_{1}\right)+b=p_{1}, \\
& \boldsymbol{w}^{T} \boldsymbol{\varphi}\left(t_{N}\right)+b=q_{1} .
\end{array}
$$

The same procedure can be applied to derive the Lagrangian and afterward the KKT optimality conditions. Then one can show that the solution to the problem (14) is obtained by solving the following linear system

$\left[\begin{array}{c|c|c|c}\mathcal{K}+I_{N-2} / \gamma & \boldsymbol{h}_{\boldsymbol{p}_{\mathbf{1}}} & \boldsymbol{h}_{\boldsymbol{q}_{\mathbf{1}}} & -\boldsymbol{f}_{\mathbf{2}} \\ \hline \boldsymbol{h}_{\boldsymbol{p}_{\mathbf{1}}}{ }^{T} & 1 & {\left[\Omega_{0}^{0}\right]_{N, 1}} & 1 \\ \hline \boldsymbol{h}_{\boldsymbol{q}_{\mathbf{1}}}{ }^{T} & {\left[\Omega_{0}^{0}\right]_{1, N}} & 1 & 1 \\ \hline-\boldsymbol{f}_{\mathbf{2}}{ }^{T} & 1 & 1 & 0\end{array}\right]\left[\begin{array}{c}\boldsymbol{\alpha} \\ \hline \beta_{1} \\ \hline \beta_{2} \\ \hline b\end{array}\right]=\left[\begin{array}{c}\boldsymbol{r} \\ \hline \frac{p_{1}}{q_{1}} \\ \hline 0\end{array}\right]$

where

$$
\begin{aligned}
& \boldsymbol{\alpha}=\left[\alpha_{2}, \ldots, \alpha_{N-1}\right]^{T}, \boldsymbol{f}_{\mathbf{1}}=\left[f_{1}\left(t_{2}\right), \ldots, f_{1}\left(t_{N-1}\right)\right]^{T} \in \mathbb{R}^{N-2}, \\
& \boldsymbol{f}_{\mathbf{2}}=\left[f_{2}\left(t_{2}\right), \ldots, f_{2}\left(t_{N-1}\right)\right]^{T} \in \mathbb{R}^{N-2}, \\
& \boldsymbol{r}=\left[r\left(t_{2}\right), \ldots, r\left(t_{N-1}\right)\right]^{T} \in \mathbb{R}^{N-2}, \\
& \mathcal{K}=\tilde{\Omega}_{2}^{2}-D_{1} \tilde{\Omega}_{2}^{1}-D_{2} \tilde{\Omega}_{2}^{0}-\tilde{\Omega}_{1}^{2} D_{1}-\tilde{\Omega}_{0}^{2} D_{2} \\
& \quad+D_{1} \tilde{\Omega}_{1}^{1} D_{1}+D_{1} \tilde{\Omega}_{0}^{1} D_{2}+D_{2} \tilde{\Omega}_{1}^{0} D_{1}+D_{2} \tilde{\Omega}_{0}^{0} D_{2}, \\
& \boldsymbol{h}_{\boldsymbol{p}_{\mathbf{1}}}=\left[\Omega_{0}^{2}\right]_{1,2: N-1}^{T}-D_{1}\left[\Omega_{0}^{1}\right]_{1,2: N-1}^{T}-D_{2}\left[\Omega_{0}^{0}\right]_{1,2: N-1}^{T}, \\
& \boldsymbol{h}_{\boldsymbol{q}_{1}}=\left[\Omega_{0}^{2}\right]_{N, 2: N-1}^{T}-D_{1}\left[\Omega_{0}^{1}\right]_{N, 2: N-1}^{T}-D_{2}\left[\Omega_{0}^{0}\right]_{N, 2: N-1}^{T} .
\end{aligned}
$$

$D_{1}$ and $D_{2}$ are diagonal matrices with the elements of $f_{1}$ and $f_{2}$ on the main diagonal respectively. Note that $\mathcal{K} \in \mathbb{R}^{(N-2) \times(N-2)}$ and $\boldsymbol{h}_{\boldsymbol{p}_{\mathbf{1}}}, \boldsymbol{h}_{\boldsymbol{q}_{\mathbf{1}}} \in \mathbb{R}^{N-2}$. $\left[\Omega_{n}^{m}\right]_{1,2: N-1}=\left[\left[\Omega_{n}^{m}\right]_{1,2}, \ldots,\left[\Omega_{n}^{m}\right]_{1, N-1}\right]$ and $\left[\Omega_{n}^{m}\right]_{N, 2: N-1}=$ $\left[\left[\Omega_{n}^{m}\right]_{N, 2}, \ldots,\left[\Omega_{n}^{m}\right]_{N, N-1}\right]$ for $n=0,1$ and $m=0,1,2$. $\tilde{\Omega}_{n}^{m}=\left[\Omega_{n}^{m}\right]_{2: N-1,2: N-1}$ for $m, n=0,1,2$.

The LS-SVM model for the solution and its derivative are expressed in dual form as

$$
\begin{aligned}
\hat{y}(t)= & \sum_{i=2}^{N-1} \alpha_{i}\left(\left[\nabla_{2}^{0} K\right]\left(t_{i}, t\right)-f_{1}\left(t_{i}\right)\left[\nabla_{1}^{0} K\right]\left(t_{i}, t\right)-\right. \\
& \left.f_{2}\left(t_{i}\right)\left[\nabla_{0}^{0} K\right]\left(t_{i}, t\right)\right)+\beta_{1}\left[\nabla_{0}^{0} K\right]\left(t_{1}, t\right)+ \\
& \beta_{2}\left[\nabla_{0}^{0} K\right]\left(t_{N}, t\right)+b, \\
\frac{d \hat{y}(t)}{d t}= & \sum_{i=2}^{N-1} \alpha_{i}\left(\left[\nabla_{2}^{1} K\right]\left(t_{i}, t\right)-f_{1}\left(t_{i}\right)\left[\nabla_{1}^{1} K\right]\left(t_{i}, t\right)-\right. \\
& \left.f_{2}\left(t_{i}\right)\left[\nabla_{0}^{1} K\right]\left(t_{i}, t\right)\right)+\beta_{1}\left[\nabla_{0}^{1} K\right]\left(t_{1}, t\right)+ \\
& \beta_{2}\left[\nabla_{0}^{1} K\right]\left(t_{N}, t\right) .
\end{aligned}
$$

\section{C. $m$-th order linear $O D E$}

Let us now consider the general $\mathrm{m}$-th order IVP of the following form:

$$
\begin{aligned}
& y^{(m)}(t)-\sum_{i=1}^{m} f_{i}(t) y^{(m-i)}(t)=r(t), \quad t \in[a, c] \\
& \left\{\begin{array}{l}
y(a)=p_{1}, \\
y^{(i-1)}(a)=p_{i}, \quad i=2, \ldots, m .
\end{array}\right.
\end{aligned}
$$

The approximate solution can be obtained by solving the following optimization problem,

$$
\begin{array}{cl}
\underset{\boldsymbol{w}, b, \boldsymbol{e}}{\operatorname{minimize}} & \frac{1}{2} \boldsymbol{w}^{T} \boldsymbol{w}+\frac{\gamma}{2} \boldsymbol{e}^{T} \boldsymbol{e} \\
\text { subject to } & \boldsymbol{w}^{T} \boldsymbol{\varphi}^{(m)}\left(t_{i}\right)=\boldsymbol{w}^{T}\left[\sum_{k=1}^{m} f_{k}\left(t_{i}\right) \boldsymbol{\varphi}_{i}^{(m-k)}\right] \\
& +f_{m}\left(t_{i}\right) b+r\left(t_{i}\right)+e_{i}, \quad i=2, \ldots, N, \\
& \boldsymbol{w}^{T} \boldsymbol{\varphi}\left(t_{1}\right)+b=p_{1}, \\
& \boldsymbol{w}^{T} \boldsymbol{\varphi}^{(i-1)}\left(t_{1}\right)=p_{i}, \quad i=2, \ldots, m .
\end{array}
$$

Lemma III.3. Given a positive definite kernel function $K$ : $\mathbb{R} \times \mathbb{R} \rightarrow \mathbb{R}$ with $K(t, s)=\varphi(t)^{T} \boldsymbol{\varphi}(s)$ and a regularization 
constant $\gamma \in \mathbb{R}^{+}$, the solution to (16) is obtained by solving the following dual problem:

$$
\left[\begin{array}{c|c|c}
\mathcal{K}+I_{N-1} / \gamma & \mathcal{K}_{i n} & -\boldsymbol{f}_{\boldsymbol{m}} \\
\hline \mathcal{K}_{\text {in }}^{T} & \Delta & C \\
\hline-\boldsymbol{f}_{\boldsymbol{m}}^{T} & C^{T} & 0
\end{array}\right]\left[\begin{array}{c}
\boldsymbol{\alpha} \\
\hline \boldsymbol{\beta} \\
\hline b
\end{array}\right]=\left[\begin{array}{c}
\boldsymbol{r} \\
\hline \boldsymbol{p} \\
\hline 0
\end{array}\right]
$$

with

$$
\begin{aligned}
& \boldsymbol{f}_{\boldsymbol{k}}=\left[f_{k}\left(t_{2}\right), \ldots, f_{k}\left(t_{N}\right)\right]^{T} \in \mathbb{R}^{N-1}, \quad k=1, \ldots, m, \\
& \boldsymbol{h}_{\boldsymbol{p}_{\boldsymbol{\ell}}}=\left[\Omega_{\ell-1}^{m}\right]_{1,2: N}^{T}-\sum_{k=1}^{m} D_{k}\left[\Omega_{\ell-1}^{m-k}\right]_{1,2: N}^{T}, \ell=1, \ldots, m \text {. } \\
& \mathcal{K}_{i n}=\left[\boldsymbol{h}_{\boldsymbol{p}_{\boldsymbol{1}}}, \ldots, \boldsymbol{h}_{\boldsymbol{p}_{m}}\right] \in \mathbb{R}^{(N-1) \times m}, \quad \boldsymbol{h}_{\boldsymbol{p}_{\boldsymbol{\ell}}} \in \mathbb{R}^{(N-1)}, \\
& \boldsymbol{p}=\left[p_{1}, \ldots, p_{m}\right]^{T} \in \mathbb{R}^{m}, \quad C=[1,0, \ldots, 0]^{T} \in \mathbb{R}^{m}, \\
& \boldsymbol{r}=\left[r\left(t_{2}\right), \ldots, r\left(t_{N}\right)\right]^{T} \in \mathbb{R}^{N-1}, \boldsymbol{\alpha}=\left[\alpha_{2}, \ldots, \alpha_{N}\right]^{T}, \\
& \boldsymbol{\beta}=\left[\beta_{1}, \ldots, \beta_{m}\right]^{T}, \bar{\Omega}_{1}=\left[\tilde{\Omega}_{m}^{0}, \ldots, \tilde{\Omega}_{m}^{m-1}\right]^{T}, \\
& \bar{D}=\left[D_{m}, \ldots, D_{1}\right], \quad \bar{\Omega}=\left[\begin{array}{ccc}
\tilde{\Omega}_{0}^{0} & \ldots & \tilde{\Omega}_{m-1}^{0} \\
\vdots & \ddots & \vdots \\
\tilde{\Omega}_{0}^{m-1} & \cdots & \tilde{\Omega}_{m-1}^{m-1}
\end{array}\right] \\
& \mathcal{K}=\tilde{\Omega}_{m}^{m}-\bar{D} \bar{\Omega}_{1}-\bar{\Omega}_{1}^{T} \bar{D}^{T}+\bar{D} \bar{\Omega} \bar{D}^{T}, \\
& \Delta=\left[\begin{array}{ccc}
{\left[\Omega_{0}^{0}\right]_{1,1}} & \cdots & {\left[\Omega_{m-1}^{0}\right]_{1,1}} \\
\vdots & \ddots & \vdots \\
{\left[\Omega_{0}^{m-1}\right]_{1,1}} & \cdots & {\left[\Omega_{m-1}^{m-1}\right]_{1,1}}
\end{array}\right]_{m \times m} .
\end{aligned}
$$

$\left\{D_{i}\right\}_{i=1}^{m}$ are diagonal matrices with the elements of $\left\{\boldsymbol{f}_{\boldsymbol{i}}\right\}_{i=1}^{m}$ on the main diagonal respectively. Also $\bar{\Omega}_{1}, \bar{\Omega}$ and $\bar{D}$ are block matrices. $\tilde{\Omega}_{n}^{m}=\left[\Omega_{n}^{m}\right]_{2: N, 2: N}$. Note that $\mathcal{K} \in \mathbb{R}^{(N-1) \times(N-1)}$.

Proof: The Lagrangian for (16) is given by

$$
\begin{aligned}
& \mathcal{L}\left(\boldsymbol{w}, b, e_{i}, \alpha_{i}, \beta_{i}\right)= \\
& \frac{1}{2} \boldsymbol{w}^{T} \boldsymbol{w}+\frac{\gamma}{2} \boldsymbol{e}^{T} \boldsymbol{e}-\sum_{i=2}^{N} \alpha_{i}\left[\boldsymbol { w } ^ { T } \left(\boldsymbol{\varphi}^{m}\left(t_{i}\right)-\sum_{k=1}^{m} f_{k}\left(t_{i}\right)\right.\right. \\
& \left.\left.\boldsymbol{\varphi}^{(m-k)}\left(t_{i}\right)\right)-f_{m}\left(t_{i}\right) b-r_{i}-e_{i}\right]-\beta_{1}\left(\boldsymbol{w}^{T} \boldsymbol{\varphi}\left(t_{1}\right)+b-p_{1}\right) \\
& -\beta_{2}\left(\boldsymbol{w}^{T} \boldsymbol{\varphi}^{\prime}\left(t_{1}\right)-p_{2}\right)-\cdots-\beta_{m}\left(\boldsymbol{w}^{T} \boldsymbol{\varphi}^{m-1}\left(t_{1}\right)-p_{m}\right) .
\end{aligned}
$$

Eliminating $\boldsymbol{w}$ and $\left\{e_{i}\right\}_{i=2}^{N}$ from the corresponding KKT optimality conditions yields the following set of equations

$$
\left\{\begin{aligned}
r_{i}= & \sum_{j=2}^{N} \alpha_{j}\left[\left[\Omega_{m}^{m}\right]_{j, i}\right. \\
& -\sum_{\ell=1}^{m} f_{\ell}\left(t_{i}\right)\left(\left[\Omega_{m}^{m-\ell}\right]_{j, i}-\sum_{k=1}^{m} f_{k}\left(t_{j}\right)\left[\Omega_{m-k}^{m-\ell}\right]_{j, i}\right) \\
& -\sum_{k=1}^{m} f_{k}\left(t_{j}\right)\left[\Omega_{m-k}^{m}\right]_{j, i} \\
& +\sum_{\ell=1}^{m} \beta_{\ell}\left(\left[\Omega_{\ell-1}^{m}\right]_{1, i}-\sum_{k=1}^{m} f_{k}\left(t_{i}\right)\left[\Omega_{\ell-1}^{m-k}\right]_{1, i}\right) \\
& +\frac{\alpha_{i}}{\gamma}-f_{m}\left(t_{i}\right) b, \quad i=2, \ldots, N
\end{aligned}\right.
$$

$$
\left\{\begin{aligned}
p_{1}= & \sum_{j=2}^{N} \alpha_{j}\left(\left[\Omega_{m}^{0}\right]_{j, 1}-\sum_{k=1}^{m} f_{k}\left(t_{j}\right)\left[\Omega_{m-k}^{0}\right]_{j, 1}\right) \\
& +\sum_{k=1}^{m} \beta_{k}\left[\Omega_{k-1}^{0}\right]_{1,1}+b, \\
\vdots & \\
p_{m}= & \sum_{j=2}^{N} \alpha_{j}\left(\left[\Omega_{m}^{m-1}\right]_{j, 1}-\sum_{k=1}^{m} f_{k}\left(t_{j}\right)\left[\Omega_{m-k}^{m-1}\right]_{j, 1}\right) \\
& +\sum_{k=1}^{m} \beta_{k}\left[\Omega_{k-1}^{m-1}\right]_{1,1}, \\
0= & \sum_{j=2}^{N} \alpha_{j} f_{m}\left(t_{j}\right)-\beta_{1} .
\end{aligned}\right.
$$

Rewriting the above system in matrix form will result in (17).

The LS-SVM model for the solution and its derivatives can be expressed in dual form as follows

$$
\begin{aligned}
\hat{y}(t)= & \sum_{i=2}^{N} \alpha_{i}\left(\left[\nabla_{m}^{0} K\right]\left(t_{i}, t\right)-\sum_{k=1}^{m} f_{k}\left(t_{i}\right)\left[\nabla_{m-k}^{0} K\right]\left(t_{i}, t\right)\right) \\
& +\sum_{k=1}^{m} \beta_{k}\left[\nabla_{k-1}^{0} K\right]\left(t_{1}, t\right)+b \\
\frac{d^{p} \hat{y}(t)}{d t^{p}}= & \sum_{i=2}^{N} \alpha_{i}\left(\left[\nabla_{m}^{p} K\right]\left(t_{i}, t\right)-\sum_{k=1}^{m} f_{k}\left(t_{i}\right)\left[\nabla_{m-k}^{p} K\right]\left(t_{i}, t\right)\right) \\
& +\sum_{k=1}^{m} \beta_{k}\left[\nabla_{k-1}^{p} K\right]\left(t_{1}, t\right) \quad, \quad p=1, \ldots, m-1 .
\end{aligned}
$$

Lemma III.4. The matrix $\mathcal{K}$ is positive semi-definite.

Proof: Let $\tilde{D}=\left[\bar{D},-I_{N-1}\right]$ and

$$
\tilde{\Omega}=\left[\begin{array}{cc}
\bar{\Omega} & \bar{\Omega}_{1} \\
\bar{\Omega}_{1}^{T} & \Omega_{m}^{m}
\end{array}\right] .
$$

Then the matrix $\mathcal{K}$ can be written as $\tilde{D} \tilde{\Omega} \tilde{D}^{T}$. To show that $x^{T} \mathcal{K} x \geq 0$ for any $x$, it is sufficient to show that $\tilde{x}^{T} \tilde{\Omega} \tilde{x} \geq$ 0 for any $\tilde{x}$ because $x=\tilde{D} \tilde{x}$ is included as a special case. Now consider matrices of evaluations of the feature map and its derivatives $\Phi^{(i)}=\left[\boldsymbol{\varphi}^{(i)}\left(t_{2}\right), \ldots, \varphi^{(i)}\left(t_{N}\right)\right]$ for $i=0, \ldots, m-1$ and denote their concatenation as $\tilde{\Phi}=\left[\Phi^{(0)}, \ldots, \Phi^{(m-1)}\right]$. Then $\|\tilde{\Phi} \tilde{x}\|_{2}^{2}=\tilde{x}^{T} \tilde{\Phi}^{T} \tilde{\Phi} \tilde{x}=\tilde{x}^{T} \tilde{\Omega} \tilde{x}$ holds, where the last equality follows from an application of the kernel trick. With the property that the norm of any vector is a non negative real number, $\|\tilde{\Phi} \tilde{x}\|_{2}$ is greater than or equal to zero. Therefore also its squared form $\tilde{x}^{T} \tilde{\Omega} \tilde{x}$ is non-negative, which concludes the proof.

\section{FORMULATION OF THE METHOD FOR THE NONLINEAR ODE CASE}

In this section we formulate an optimization problem based on least squares support vector machines for solving nonlinear first order ordinary differential equations of the following form

$$
y^{\prime}=f(t, y), \quad y(a)=p_{1}, \quad a \leq t \leq c .
$$

One starts with assuming the approximate solution to be of the form $\hat{y}(t)=\boldsymbol{w}^{T} \boldsymbol{\varphi}(t)+b$. Additional unknowns $y_{i}$ are introduced to keep the constraints linear in $\boldsymbol{w}$. This yields the following nonlinear optimization problem: 


$$
\begin{array}{cl}
\underset{\boldsymbol{w}, b, \boldsymbol{e}, \boldsymbol{\xi}, y_{i}}{\operatorname{minimize}} & \frac{1}{2} \boldsymbol{w}^{T} \boldsymbol{w}+\frac{\gamma}{2} \boldsymbol{e}^{T} \boldsymbol{e}+\frac{\gamma}{2} \boldsymbol{\xi}^{T} \boldsymbol{\xi} \\
\text { subject to } & \boldsymbol{w}^{T} \boldsymbol{\varphi}^{\prime}\left(t_{i}\right)=f\left(t_{i}, y_{i}\right)+e_{i}, \quad i=2, \ldots, N, \\
& \boldsymbol{w}^{T} \boldsymbol{\varphi}\left(t_{1}\right)+b=p_{1}, \\
& y_{i}=\boldsymbol{w}^{T} \boldsymbol{\varphi}\left(t_{i}\right)+b+\xi_{i}, \quad i=2, \ldots, N .
\end{array}
$$

The Lagrangian of the constrained optimization problem (19) becomes

$$
\begin{aligned}
& \mathcal{L}\left(\boldsymbol{w}, b, e_{i}, \xi_{i}, y_{i}, \alpha_{i}, \eta_{i}, \beta\right)= \\
& \frac{1}{2} \boldsymbol{w}^{T} \boldsymbol{w}+\frac{\gamma}{2} \boldsymbol{e}^{T} \boldsymbol{e}+\frac{\gamma}{2} \boldsymbol{\xi}^{T} \boldsymbol{\xi}-\sum_{i=2}^{N} \alpha_{i}\left(\boldsymbol{w}^{T} \boldsymbol{\varphi}^{\prime}\left(t_{i}\right)-\right. \\
& \left.f\left(t_{i}, y_{i}\right)-e_{i}\right)-\beta\left(\boldsymbol{w}^{T} \boldsymbol{\varphi}\left(t_{1}\right)+b-p_{1}\right)- \\
& \sum_{i=2}^{N} \eta_{i}\left(y_{i}-\boldsymbol{w}^{T} \boldsymbol{\varphi}\left(t_{i}\right)-b-\xi_{i}\right) .
\end{aligned}
$$

After obtaining KKT optimality conditions, and elimination of the primal variables $\boldsymbol{w},\left\{e_{i}\right\}_{i=2}^{N}$ and $\left\{\xi_{i}\right\}_{i=2}^{N}$ and making use of Mercer's Theorem, the solution is obtained in the dual by solving the following nonlinear system of equations

$\left[\begin{array}{c|c|c|c|c}\widehat{\Omega_{1}^{1}} & \tilde{\Omega}_{0}^{1} & \boldsymbol{h}_{\mathbf{1}}^{T} & \mathbf{0}_{N-1} & 0_{(N-1) \times(N-1)} \\ \hline\left(\tilde{\Omega}_{0}^{1}\right)^{T} & \widehat{\Omega}_{0}^{0} & \boldsymbol{h}_{\mathbf{0}}^{T} & \mathbf{1}_{N-1} & -I_{N-1} \\ \hline \boldsymbol{h}_{\mathbf{1}} & \boldsymbol{h}_{\mathbf{0}} & {\left[\Omega_{0}^{0}\right]_{1,1}} & 1 & \mathbf{0}_{N-1}^{T} \\ \hline \mathbf{0}_{N-1}^{T} & \mathbf{1}_{N-1}^{T} & 1 & 0 & \mathbf{0}_{N-1}^{T} \\ \hline D(\boldsymbol{y}) & I_{N-1} & \mathbf{0}_{N-1} & \mathbf{0}_{N-1} & 0_{(N-1) \times(N-1)}\end{array}\right]$

$$
\left[\begin{array}{l}
\boldsymbol{\alpha} \\
\hline \boldsymbol{\eta} \\
\hline \beta \\
\hline b \\
\hline \boldsymbol{y}
\end{array}\right]=\left[\begin{array}{l}
\boldsymbol{f}(\boldsymbol{y}) \\
\hline \mathbf{0}_{N-1} \\
\hline p_{1} \\
\hline 0 \\
\hline \mathbf{0}_{N-1}
\end{array}\right]
$$

where

$$
\begin{aligned}
& \widehat{\Omega}_{1}^{1}=\tilde{\Omega}_{1}^{1}+I_{N-1} / \gamma, \quad \widehat{\Omega}_{0}^{0}=\tilde{\Omega}_{0}^{0}+I_{N-1} / \gamma, \\
& D(\boldsymbol{y})=\operatorname{diag}\left(\boldsymbol{f}^{\prime}(\boldsymbol{y})\right) \\
& \boldsymbol{f}(\boldsymbol{y})=\left[f\left(t_{2}, y_{2}\right), \ldots, f\left(t_{N}, y_{N}\right)\right]^{T}, \\
& \boldsymbol{f}^{\prime}(\boldsymbol{y})=\left[\left.\frac{\partial f(t, y)}{\partial y}\right|_{t=t_{2}, y=y_{2}}, \ldots,\left.\frac{\partial f(t, y)}{\partial y}\right|_{t=t_{N}, y=y_{N}}\right], \\
& \boldsymbol{\alpha}=\left[\alpha_{2}, \ldots, \alpha_{N}\right]^{T}, \boldsymbol{\eta}=\left[\eta_{2}, \ldots, \eta_{N}\right]^{T}, \\
& \boldsymbol{y}=\left[y_{2}, \ldots, y_{N}\right]^{T}, \tilde{\Omega}_{0}^{0}=\left[\Omega_{0}^{0}\right]_{2: N, 2: N} \\
& \tilde{\Omega}_{1}^{1}=\left[\Omega_{1}^{1}\right]_{2: N, 2: N}, \tilde{\Omega}_{0}^{1}=\left[\Omega_{0}^{1}\right]_{2: N, 2: N} \\
& \boldsymbol{h}_{\mathbf{0}}=\left[\left[\Omega_{0}^{0}\right]_{1,2}, \ldots,\left[\Omega_{0}^{0}\right]_{1, N}\right] \\
& \boldsymbol{h}_{\mathbf{1}}=\left[\left[\Omega_{0}^{1}\right]_{1,2}, \ldots,\left[\Omega_{0}^{1}\right]_{1, N}\right] \\
& \mathbf{0}_{N-1}=[0, \ldots, 0]^{T} \in \mathbb{R}^{N-1} .
\end{aligned}
$$

The nonlinear system (20), which consists of $3 N-1$ equations with $3 N-1$ unknowns $(\boldsymbol{\alpha}, \boldsymbol{\eta}, \beta, b, \boldsymbol{y})$, is solved by Newton's method. The model in the dual form becomes

$$
\begin{aligned}
\hat{y}(t)= & \sum_{i=2}^{N} \alpha_{i}\left[\nabla_{1}^{0} K\right]\left(t_{i}, t\right)+\sum_{i=2}^{N} \eta_{i}\left[\nabla_{0}^{0} K\right]\left(t_{i}, t\right)+ \\
& \beta\left[\nabla_{0}^{0} K\right]\left(t_{1}, t\right)+b .
\end{aligned}
$$

\section{PRACTICAL IMPLEMENTATION AND MODEL SELECTION}

\section{A. Solution on long time Interval}

Consider now the situation where a given differential equation has to be solved for a large time interval $[a, c]$. It should be noted that in order to improve the accuracy (or maintain the same order of accuracy on the whole domain) we then need to increase the number of collocation points. This approach however leads to a larger system of equations.

In order to implement the proposed method for solving problems involving large time intervals efficiently, the domain decomposition technique is applied [25]. At first the domain $\Xi=[a, c]$ is decomposed into $S$ segments as $\Xi=\bigcup_{k=1}^{S} \Xi_{k}$. We assume that the approximate solution on the $k$-th segment has the form $\hat{y}_{k}(t)=\boldsymbol{w}_{k}^{T} \boldsymbol{\varphi}(t)+b_{k}$. Then the problem is solved in each sub-domain $\Xi_{k}$ using the described method in previous sections. The computed approximate solution at the final point in the sub-domain $\Xi_{k}$ is used as starting point (initial value) for the consecutive sub-domain $\Xi_{k+1}$.

Utilizing this approach will result in solving $S$ smaller systems of equations, which is computationally more efficient than solving a very large system of equations obtained by considering the whole domain $\Xi$ (with the same total number of collocation points). The procedure is outlined in Algorithm 1.

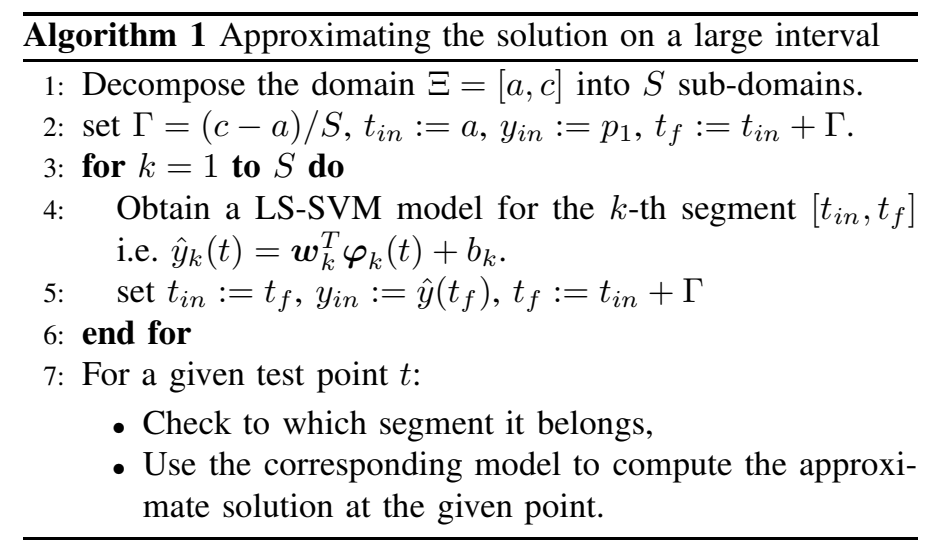

\section{B. Parameter tuning}

The performance of the LS-SVM model depends on the choice of the tuning parameters. In this paper for all experiments the Gaussian RBF kernel is used. Therefore a model is determined by the regularization parameter $\gamma$ and the kernel bandwidth $\sigma$.

It should be noted that unlike the regression case, we do not have target values and consequently we do not have noise. Therefore a quite large value should be taken for the regularization constant $\gamma$ so that the error $e$ is sharply minimized or equivalently the constraints are well satisfied. 
In all the experiments the chosen value for $\gamma$ was $10^{10}$, except for the problem with large interval for which $\gamma$ is set to $10^{5}$ in order to avoid ill conditioning.

Therefore the only parameter left that has to be tuned is the kernel bandwidth. In this work, the optimal values of $\sigma$ are obtained by evaluating the performance of the model on a validation set using a meaningful range of possible $(\sigma)$. The validation set is defined to be the set of midpoints $V \equiv\left\{v_{i}=\right.$ $\left.\frac{\left(t_{i}+t_{i+1}\right)}{2}, i=1, \ldots, N-1\right\}$ where $\left\{t_{i}\right\}_{i=1}^{N}$ are training points. The values that minimize the mean squared error (MSE) on this validation set are then selected.

Remark V.1. In some cases, an extremely large value for $\gamma$, normally greater than $10^{7}$, can make the matrix in (9) close to singular.

\section{Numerical Results}

In this section, we have tested the performance of the proposed method on seven problems, four first order and three second order ODEs. For the first three problems and problem 5.5, a comparison is made between the solutions obtained in [17] and our computed solutions. The numerical results of the problems 5.4 and 5.6 are compared with those given in [16]. Problem 5.7 which has no analytic solution and is a singular problem is solved and the computed solution is compared with that reported in [26]. In order to show the approximation and generalization capabilities of the proposed method, we compare the exact solution with the computed solution inside and outside of the domain of consideration. Furthermore the proposed method is successfully applied to solve problem 5.1 for a very large time interval. For all the experiments, the RBF kernel is used, $K(u, v)=\exp \left(-\frac{(u-v)^{2}}{\sigma^{2}}\right)$, so the following relations hold,

$$
\begin{aligned}
& \nabla_{1}^{0}[K(u, v)]=-\frac{2(u-v)}{\sigma^{2}} K(u, v), \\
& \nabla_{0}^{1}[K(u, v)]=\frac{2(u-v)}{\sigma^{2}} K(u, v), \\
& \nabla_{2}^{0}[K(u, v)]=\left[\frac{4(u-v)^{2}}{\sigma^{4}}-\frac{2}{\sigma^{2}}\right] K(u, v) .
\end{aligned}
$$

Matlab 2010b is used to implement the code and all computations were carried out on a windows 7 system with Intel(R)core(TM) i7 CPU and 4.00GB RAM.

\section{A. First order ODEs}

Problem 5.1: Consider the following first order ODE [17, Example 2]:

$$
\frac{d}{d t} y(t)+2 y(t)=\sin (t), \quad y(0)=1, \quad t \in[0,10] .
$$

The approximate solution obtained by the proposed method is compared with the true solution and results are depicted in Fig 1. From the obtained results, it is apparent that our method outperforms the method in [17] in terms of accuracy (see [17, Fig 6]), although training was performed using much less number of points (one fourth). In addition we also considered points outside the training interval, and Fig 1 (d) and (e) show that the extrapolation error remains low for the points near the domain of equation. As it was expected by increasing the number of mesh points (training points), the error decreases both inside and outside of the training interval. Fig 1 (c) and (f) indicate the performance of the method when non-uniform partitioning is used for creating training points.

Problem 5.2: First order differential equation with nonlinear sinusoidal excitation [17, Example 3] :

$$
\frac{d}{d t} y(t)+2 y(t)=t^{3} \sin (t / 2), \quad y(0)=1, \quad t \in[0,10] .
$$

The interval $[0,10]$ is discretized into $N=20$ points $t_{1}=0, \ldots, t_{20}=10$ using the grid $t_{i}=(i-1) h, i=1, \ldots, N$, where $h=\frac{10}{N-1}$. In Fig 2(a), we compare the exact solution with the computed solution at grid points (circles) as well as for other points inside and outside the domain of equation. The obtained absolute errors for points inside and outside the domain $[0,10]$ are tabulated in Table I. It is clear that the solution is of higher accuracy compared to the solution obtained in [17] despite the fact that much less number of training points are used. (Note that in [17], 100 equidistant points are used for training and the maximum absolute error shown in [17, Fig 13] is approximately $25 \times 10^{-2}$ ).

Problem 5.3: Consider the following nonlinear first order ODE which has no analytic solution [17, Example 6]:

$$
\frac{d}{d t} y(t)=y(t)^{2}+t^{2}, \quad y(0)=1, \quad t \in[0,0.5] .
$$

Twenty equidistant points in the given interval are used for the training process. The obtained approximate solution by the proposed method and the solution obtained by MATLAB built-in solver ode45 are displayed in Fig 2(b). The obtained absolute errors for points inside and outside the domain [0,0.5] are tabulated in Table I. The proposed method shows a better performance in comparison with the described method in [17] in terms of accuracy despite the fact that much less number of training points are used. (Note that in [17] the problem is solved over domain $[0,0.2]$ by using 100 equidistant training points and the maximum absolute error shown in [17, Fig 22] is approximately $4 \times 10^{-2}$ ).

Problem 5.4: Consider the following first order ODE with time varying coefficient [16, Problem 1]:

$$
\begin{aligned}
& \frac{d}{d t} y(t)+\left(t+\frac{1+3 t^{2}}{1+t+t^{3}}\right) y(t)=t^{3}+2 t+t^{2} \frac{1+3 t^{2}}{1+t+t^{3}}, \\
& y(0)=1, \quad t \in[0,1] .
\end{aligned}
$$

In order to have a fair comparison with the results reported in [16], ten equidistant points in the given interval are used for the training process. The analytic solution and obtained solution via our proposed method are displayed in Fig 2(c). The obtained absolute errors for points inside and outside the domain $[0,1]$ are recorded in Table I, which shows the superiority of the proposed method over the described method in [16]. (Note that in [16, Fig 2] the maximum absolute error outside the domain $[0,1]$ is approximately $12 \times 10^{-2}$ ). 


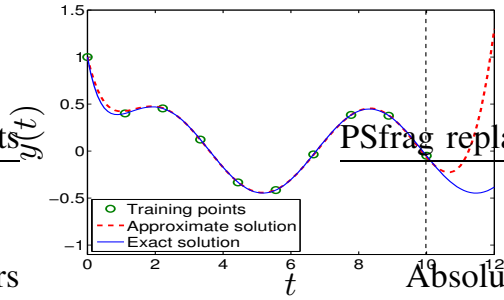

(a)

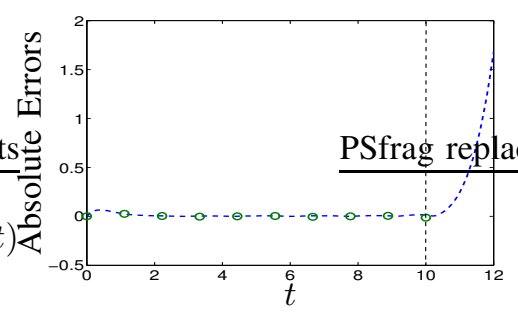

(d)

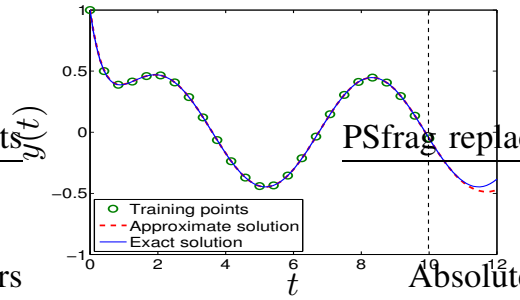

(b)

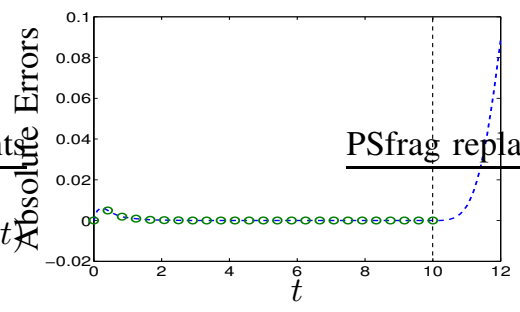

(e)

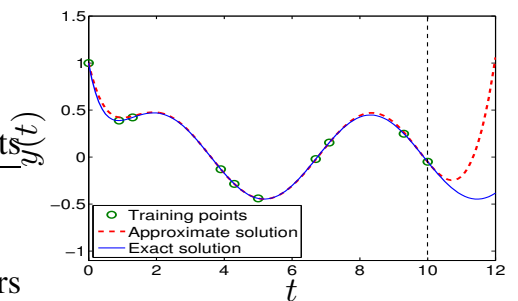

(c)

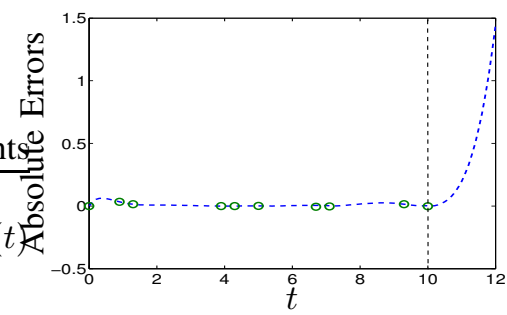

(f)

Fig. 1. Numerical results for Problem 5.1. (a) 10 equidistant points in $[0,10]$ are used for training. (b) 25 equidistant points in $[0,10]$ are used for training. (c) Non-uniform partitions of $[0,10]$ using 10 points which are used for training. (d) Obtained absolute errors on the interval $[0,12]$ when $[0,10]$ is discretized into 9 equal parts. (e) Obtained absolute errors on the interval $[0,12]$ when $[0,10]$ is discretized into 24 equal parts. (f) Obtained absolute errors on the interval $[0,12]$ when $[0,10]$ is discretized into 9 non-uniform parts.

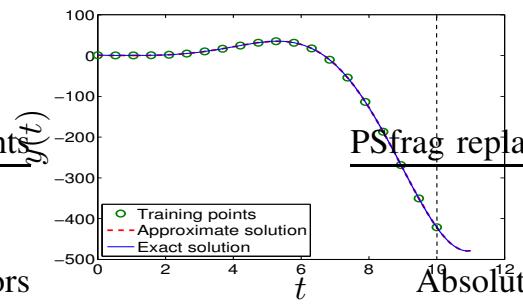

(a)

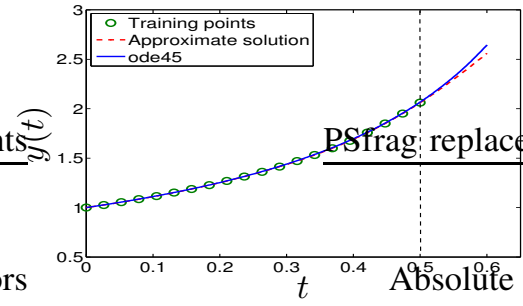

(b)

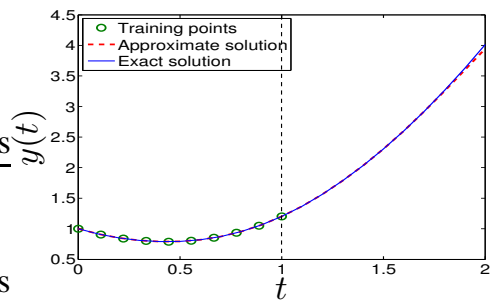

(c)

Fig. 2. (a) Numerical results for Problem 5.2. Twenty equidistant points in $[0,10]$ are used for training. (b) Numerical results for Problem 5.3. Twenty equidistant points in $[0,0.5]$ are used for training. (c) Numerical results for Problem 5.4. Ten equidistant points in $[0,1]$ are used for training.

\section{B. Second order ODEs}

Problem 5.5: Consider the following second order boundary value problem with time-varying input signal [17, Example 4]:

$$
\begin{aligned}
& \frac{d^{2}}{d t^{2}} y(t)+y(t)=2+2 \sin (4 t) \cos (3 t), \\
& y(0)=1, \quad y(1)=0 .
\end{aligned}
$$

Ten equidistant points in the given interval are used for the training process. The analytic solution and the obtained solution via our proposed method are displayed in Fig 3(a). The obtained absolute errors for points inside and outside the domain $[0,1]$ are recorded in Table II which shows the superiority of the proposed method over the described method in [17]. (Note that in [17], 100 equidistant points are used for training and the maximum absolute error shown in [17, Fig 17] is approximately $5 \times 10^{-1}$ ).

Problem 5.6: Consider the following second order ODE with time-varying input signal [16, Problem 3] :

$$
\begin{aligned}
& \frac{d^{2}}{d t^{2}} y(t)+\frac{1}{5} \frac{d}{d t} y(t)+y(t)=-\frac{1}{5} e^{(-t / 5)} \cos (t), \\
& y(0)=1, \quad y^{\prime}(0)=1 .
\end{aligned}
$$

Ten equidistant points in the interval $[0,2]$ are used for the training process. The analytic solution and the obtained solution by the proposed method are shown in Fig 3(b). The obtained absolute errors for points inside and outside the domain [0,2] are tabulated in Table II, which again shows the improvement of the proposed method over the described method in [16]. (Note that in [16, Fig 4] the maximum absolute error outside the domain $[0,2]$ is $8 \times 10^{-4}$ ).

Problem 5.7: Consider the following singular second order ODE which has no analytical closed form solution [26, Example 1]:

$$
\begin{aligned}
& \frac{d^{2}}{d t^{2}} y(t)+\frac{1}{t} \frac{d}{d t} y(t)-\frac{1}{t} \cos (t)=0, \quad y(0)=0, \quad y^{\prime}(0)=1 . \\
& \text { Exact solution: } y(t)=\int_{0}^{t} \frac{\sin (x)}{x} d x .
\end{aligned}
$$

Ten equidistant points in the interval $[0,1]$ are used as training points and the obtained result are shown in Fig 3(c) and recorded in Table II. The obtained maximum absolute error outside the domain $[0,1]$ is $6.51 \times 10^{-2}$ which is smaller than $14 \times 10^{-1}$ shown in [26, Fig 6]. 


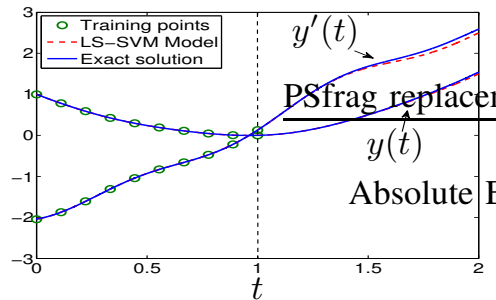

(a)

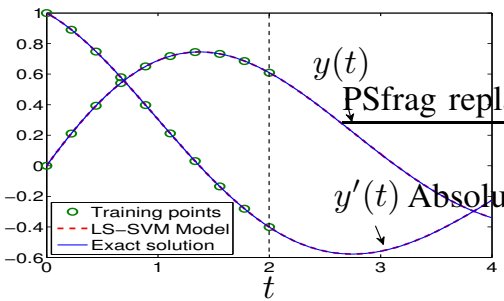

(b)

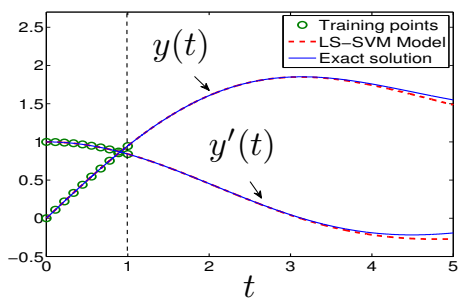

(c)

Fig. 3. (a) Numerical results for Problem 5.5. Ten equidistant points in [0,1] are used for training, (b) Numerical results for Problem 5.6. Ten equidistant points in $[0,2]$ are used for training. (c) Numerical results for Problem 5.7. Ten equidistant points in $[0,1]$ are used for training.

TABLE I

Numerical Results of The PRoposed Method FOR SOlving PROBlems 5.2, 5.3 AND 5.4.

\begin{tabular}{ccccc}
\hline Problem & Domain & $\|\boldsymbol{y}-\hat{\boldsymbol{y}}\|_{\boldsymbol{\infty}}$ & MSE & STD \\
\hline \multirow{2}{*}{5.2} & Inside & $4.56 \times 10^{-3}$ & $1.47 \times 10^{-6}$ & $1.16 \times 10^{-3}$ \\
& Outside & $4.62 \times 10^{-1}$ & $3.85 \times 10^{-2}$ & $1.56 \times 10^{-1}$ \\
\hline \multirow{2}{*}{5.3} & Inside & $5.43 \times 10^{-3}$ & $8.94 \times 10^{-6}$ & $1.60 \times 10^{-3}$ \\
& Outside & $8.46 \times 10^{-2}$ & $1.49 \times 10^{-3}$ & $2.27 \times 10^{-2}$ \\
\hline \multirow{2}{*}{5.4} & Inside & $1.46 \times 10^{-4}$ & $8.15 \times 10^{-9}$ & $3.90 \times 10^{-5}$ \\
& Outside & $6.76 \times 10^{-2}$ & $5.53 \times 10^{-4}$ & $2.20 \times 10^{-2}$ \\
\hline
\end{tabular}

Note: MSE is the mean squared error and STD is the stand deviation.

TABLE II

NumericAl RESUlts of THE PROPOSED METHOD FOR SOLVING PROBLEMS 5.5, 5.6 AND 5.7.

\begin{tabular}{cccccc}
\hline Problem & Domain & Variable & $\|\boldsymbol{y}-\hat{\boldsymbol{y}}\|_{\infty}$ & MSE & STD \\
\hline 5.5 & Inside & $y$ & $1.14 \times 10^{-6}$ & $4.16 \times 10^{-13}$ & $6.43 \times 10^{-7}$ \\
& & $y^{\prime}$ & $4.81 \times 10^{-5}$ & $6.78 \times 10^{-11}$ & $8.21 \times 10^{-6}$ \\
& Outside & $y$ & $4.20 \times 10^{-2}$ & $2.64 \times 10^{-4}$ & $1.26 \times 10^{-2}$ \\
& & $y^{\prime}$ & $1.00 \times 10^{-1}$ & $3.27 \times 10^{-3}$ & $3.87 \times 10^{-2}$ \\
\hline \multirow{2}{*}{5.6} & \multirow{2}{*}{ Inside } & $y$ & $5.88 \times 10^{-6}$ & $1.49 \times 10^{-11}$ & $1.63 \times 10^{-6}$ \\
& & $y^{\prime}$ & $7.34 \times 10^{-6}$ & $2.18 \times 10^{-11}$ & $3.28 \times 10^{-6}$ \\
& \multirow{2}{*}{ Outside } & $y$ & $3.96 \times 10^{-4}$ & $2.39 \times 10^{-8}$ & $1.19 \times 10^{-4}$ \\
& & $y^{\prime}$ & $5.15 \times 10^{-4}$ & $7.11 \times 10^{-8}$ & $1.74 \times 10^{-4}$ \\
\hline \multirow{2}{*}{5.7} & \multirow{2}{*}{ Inside } & $y$ & $6.64 \times 10^{-9}$ & $2.01 \times 10^{-16}$ & $4.07 \times 10^{-9}$ \\
& & $y^{\prime}$ & $8.41 \times 10^{-8}$ & $1.30 \times 10^{-15}$ & $3.59 \times 10^{-8}$ \\
& \multirow{2}{*}{ Outside } & $y$ & $6.51 \times 10^{-2}$ & $3.90 \times 10^{-4}$ & $1.65 \times 10^{-2}$ \\
& & $y^{\prime}$ & $7.80 \times 10^{-2}$ & $7.31 \times 10^{-4}$ & $2.15 \times 10^{-2}$ \\
\hline \multicolumn{4}{r}{ Note: } & MSE is the mean squared error and STD is the stand deviation.
\end{tabular}

\section{Sensitivity of the solution w.r.t the parameter}

In order to illustrate the sensitivity of the result with respect to the parameter of the model $(\sigma)$, for two examples we have plotted the MSE, on the validation set, versus the kernel bandwidth on logarithmic scales in Fig 4. From this figure, it is apparent that there exist a range of $\sigma$ for which the MSE on the validation set is quite small.

\section{Large interval}

Let us consider problem 5.1 when the time interval is $\left[0,10^{5}\right]$. It is known in advance that the solution of this problem is oscillating. The problem is solved by decomposing the given domain of interest into $S$ sub-domains. Then the problem is solved on each sub-domain using $N$ number of local collocation points. The execution time and the mean square error (MSE) for

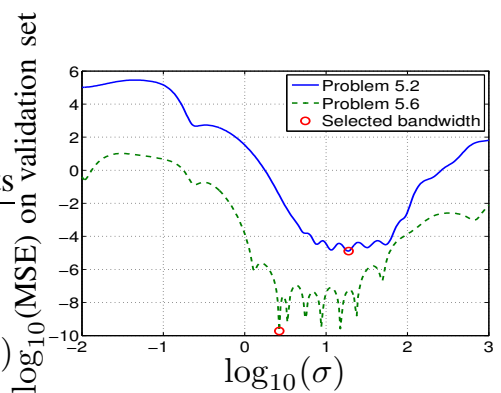

Fig. 4. Sensitivity of the obtained result with respect to model parameter $\sigma$. $\log _{10}$ (MSE) vs. $\log _{10} \sigma$ is plotted for Problems 5.2 and 5.6.

the training and test sets

$$
\begin{aligned}
M S E_{\text {train }} & =\frac{\sum_{i=1}^{N \times S}\left(y\left(t_{i}\right)-\hat{y}\left(t_{i}\right)\right)^{2}}{N \times S}, \\
M S E_{\text {test }} & =\frac{\sum_{i=1}^{M}\left(y\left(t_{i}\right)-\hat{y}\left(t_{i}\right)\right)^{2}}{M},
\end{aligned}
$$

where $N \times S$ is the total number of collocation points and $M$ is the total number of test points over the interval $\left[0,10^{5}\right]$, are tabulated in Table III. The test set is the same for all the cases and it consists of $M=5 \times 10^{5}$ points. It is apparent that when $S$ is fixed and $N$ increases, the accuracy is improved whereas the execution time is increased. The same pattern is observed when $N$ is fixed and $S$ increases. Fig 5 (a) and (b) show the residual error $e_{t}=y(t)-\hat{y}(t)$ when Problem 5.1 is solved over the interval $\left[0,10^{5}\right]$, using $N=50$ local collocation points, $S=5000$ sub-domains and $N=500$, collocation points, $S=500$ sub-domains respectively. It should be noted that the result depicted in Fig. 5(a) is obtained much faster than that shown in Fig. 5(b).

In Table IV, we analyze the situation where the total number of collocation points i.e. $N \times S$ in the given interval $[0,4000]$ is fixed. It can be seen that as the number of sub-domains increases (number of collocation points in each sub-domain increases) the computational time decreases without losing the order of accuracy. In this case the test set consists of $M=2 \times 10^{4}$ points.

\section{CONCLUSION AND FUTURE WORK}

In this paper, a new method based on least squares support vector machines is developed for solving general linear $m$-th order ODEs and also first order nonlinear ODE. On the tested problems the method proposed in this paper is more efficient compared to methods described in [16] and [17]. Also the 
TABLE III

Numerical RESUlt of THE PROPOSED METHOd FOR SOLVING PROBLEM 5.1 WITH TIME INTERVAL $\left[0,10^{5}\right] . N$ IS THE NUMBER OF LOCAL COLLOCATION POINTS AND $S$ IS THE NUMBER SUB-DOMAINS.

\begin{tabular}{lllll}
\hline \multirow{2}{*}{$N$} & \multirow{2}{*}{$S$} & CPU time & \multicolumn{2}{c}{ MSE } \\
\cline { 4 - 5 } 20 & 1000 & 5.5 & $2.4 \times 10^{-2}$ & $7.2 \times 10^{-2}$ \\
& 2000 & 10.6 & $1.3 \times 10^{-3}$ & $3.3 \times 10^{-3}$ \\
& 5000 & 29.5 & $8.4 \times 10^{-8}$ & $2.3 \times 10^{-7}$ \\
\hline \multirow{2}{*}{30} & 1000 & 6.6 & $2.2 \times 10^{-2}$ & $5.9 \times 10^{-2}$ \\
& 2000 & 13.4 & $4.1 \times 10^{-6}$ & $1.3 \times 10^{-5}$ \\
& 5000 & 37.1 & $8.2 \times 10^{-9}$ & $2.7 \times 10^{-8}$ \\
\hline \multirow{2}{*}{40} & 1000 & 9.6 & $5.8 \times 10^{-4}$ & $1.4 \times 10^{-3}$ \\
& 2000 & 20.1 & $1.7 \times 10^{-7}$ & $5.8 \times 10^{-7}$ \\
& 5000 & 54.2 & $2.3 \times 10^{-9}$ & $8.1 \times 10^{-9}$ \\
\hline
\end{tabular}

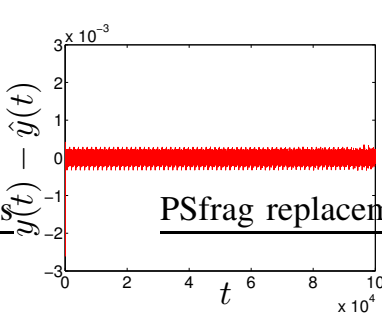

(a)

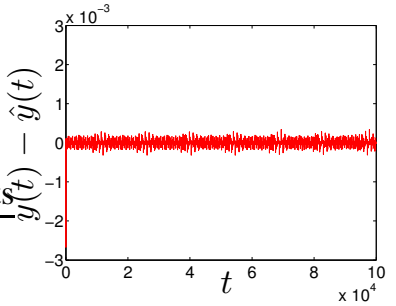

(b)
Fig. 5. (a) Residual $y(t)-\hat{y}(t)$ when problem 5.1 is solved on the interval $\left[0,10^{5}\right]$, by using 5000 sub-intervals and 50 local collocation points. (b) Obtained residual $y(t)-\hat{y}(t)$ for the same problem by using 500 sub-intervals and 500 local collocation points.

proposed method is able to solve a differential equation for a large time interval while predicting the solution with high accuracy.

The proposed method may be extended to solve a system of ordinary differential equations and partial differential equations which can be considered as future challenges.

\section{ACKNOWLEDGMENT}

This work was supported by Research Council KUL: GOA/11/05 Ambiorics, GOA/10/09 MaNet, CoE EF/05/006 Optimization in Engineering(OPTEC), IOF-SCORES4CHEM, several $\mathrm{PhD} /$ postdoc \& fellow grants;Flemish Government:FWO: $\mathrm{PhD} /$ postdoc grants, projects: G0226.06 (cooperative systems and optimization), G0321.06 (Tensors), G.0302.07 (SVM/Kernel), G.0320.08 (convex MPC), G.0558.08 (Robust MHE), G.0557.08 (Glycemia2), G.0588.09 (Brain-machine), G.0377.12 (structured models) research communities (WOG: ICCoS, ANMMM, MLDM); G.0377.09 (Mechatronics MPC) IWT: PhD Grants, Eureka-Flite+, SBO LeCoPro, SBO Climaqs, SBO POM, O\&O-Dsquare; Belgian Federal Science Policy Office: IUAP P6/04 (DYSCO, Dynamical systems, control and optimization, 2007-2011); EU: ERNSI; FP7-HD-MPC (INFSO-ICT223854), COST intelliCIS, FP7-EMBOCON (ICT-248940); Contract Research: AMINAL; Other:Helmholtz: viCERP, ACCM, Bauknecht, Hoerbiger, ERC AdG A-DATADRIVE-B. Johan Suykens is a professor at the KU Leuven, Belgium.

\section{REFERENCES}

[1] J.C. Butcher, Numerical Methods for Ordinary Differential Equations, 2nd Edition, John Wiley \& Sons, Chichester, 2008.

[2] J.D. Lambert, Numerical Methods for Ordinary Differential Systems, Wiley, New York, 1991.

[3] M.M. Chawla, C.P. Katti, "Finite difference methods for two-point boundary value problems involving high order differential equations," BIT Numerical Mathematics, 19, pp. 27-33, 1979.

[4] R.D. Russell, L.F. Shampine, "A collocation method for boundary value problems," Numer. Math, 19, pp. 1-28, 1972.
TABLE IV

NUMERICAL RESULTS OF THE PROPOSED METHOD FOR SOLVING PROBLEM 5.1 WITH TIME INTERVAL $[0,4000]$, WHILE TOTAL NUMBER OF COLLOCATION POINTS I.E. $N \times$ $S$ IS CONSTANT

\begin{tabular}{ccccc}
\hline & & & \multicolumn{2}{c}{ MSE } \\
\cline { 4 - 5 }$N$ & $S$ & CPU time & Training & Test \\
\hline 800 & 10 & 85.5 & $1.36 \times 10^{-8}$ & $2.06 \times 10^{-8}$ \\
400 & 20 & 26.1 & $1.37 \times 10^{-8}$ & $2.08 \times 10^{-8}$ \\
20 & 400 & 2.06 & $1.68 \times 10^{-8}$ & $2.52 \times 10^{-8}$ \\
\hline
\end{tabular}

[5] C.W. Gear, "Hybrid methods for initial value problems in ordinary differential equations," SIAM Journal on Numerical Analysis, 2, pp. 69-86, 1965.

[6] D. Sarafyan, "New algorithm for the continuous approximate solution of ordinary differential equations and the upgrading of the order of the processes," Computers \& Mathematics with Applications 20(1), pp. 71-100, 1990.

[7] S.N. Jator and J. Li, "A self-starting linear multistep method for a direct solution of the general second-order initial value problem," International Journal of Computer Mathematics, 86(5), pp. 827-836, 2009.

[8] D.O. Awoyemi, "A new sixth-order algorithm for general second order ordinary differential equation," International Journal of Computer Mathematics, 77, pp. 117-124, 2001.

[9] A.J. Meade, Jr. and A.A. Fernadez, "The numerical solution of Linear Ordinary Differential Equations by Feedforward Neural networks," Math. Comput. Modelling, vol. 19, no. 12, pp. 1-25, 1994.

[10] H. Lee, and I. Kang, "Neural algorithms for solving differential equations," Journal of Computational Physics, vol. 91, pp. 110-117, 1990.

[11] B.Ph. van Milligen, V. Tribaldos, and J.A. Jiménez, "Neural Network Differential Equation and Plasma Equilibrium Solver,' Phys. Rev. Lett, 75, pp. 3594-3597, 1995.

[12] L.P. Aarts, P. Van der Veer, "Solving Systems of First Order Linear Differential Equations by a Neural Network Method," Lecture Notes in Computer Science, Volume 2074/2001, 181-189, 2001.

[13] P. Ramuhalli, L. Udpa, S.S. Udpa, "Finite-element neural networks for solving differential equations," IEEE Transactions on Neural Networks, vol. 16, no. 6, pp. 1381-1392, 2005.

[14] K.S. McFall and J.R. Mahan, "Artificial Neural Network Method for Solution of Boundary Value Problems With Exact Satisfaction of Arbitrary Boundary Conditions," IEEE Transactions on Neural Networks, vol. 20, no. 8, pp. 1221-1233, 2009.

[15] I.G. Tsoulos, D. Gavrilis, E. Glavas, "Solving differential equations with constructed neural networks," Neurocomputing, 72, pp. 2385-2391, 2009.

[16] I. Lagaris, A. Likas, and D.I. Fotiadis, "Artificial neural networks for solving ordinary and partial differential equations," IEEE Transactions on Neural Networks, vol. 9, no. 5, pp. 987-1000, 1998.

[17] H.S. Yazdi, M. Pakdaman, H. Modaghegh, "Unsupervised kernel least mean square algorithm for solving ordinary differential equations," Neurocomputing, 74, pp. 2062-2071, 2011.

[18] B. Schölkopf, A. Smola, Learning with Kernels, MIT Press, Cambridge, MA, 2002.

[19] V. Vapnik, Statistical learning theory, New York: Wiley, 1998.

[20] J. A. K. Suykens, J. Vandewalle, "Least squares support vector machine classifiers", Neural Processing Letters, 9 (3), pp. 293-300, 1999.

[21] J. A. K. Suykens, T. Van Gestel, J. De Brabanter, B. De Moor, J. Vandewalle, Least Squares Support Vector Machines, World Scientific Pub. Co., Singapore, 2002.

[22] J.A.K. Suykens, J. Vandewalle, B. De Moor, "Optimal control by least squares support vector machines," Neural Networks, vol. 14, no. 1, pp 23-35, 2001.

[23] M. Lázaro, I. Santamaria, F. Pérez-Cruz, A. Artés-Rodriguez, "Support vector regression for the simultaneous learning of a multivariate function and its derivative," Neurocomputing, 69 (1-3), pp. 42-61, 2005.

[24] D.R. Kincaid, E.W. Cheney, Numerical Analysis: Mathematics of Scientific Computing, third ed., Brooks/Cole, Pacific Grove, CA, 2002.

[25] A. Toselli and O.B. Widlund, Domain Decomposition Methods- Algorithms and Theory. Springer-Verlag, Berlin and Heidelberg, 2005.

[26] I. G. Tsoulos, I. E. Lagaris, "Solving differential equations with genetic programming," Genetic Programming and Evolvable Machines, 7, pp. 3354, 2006. 